\title{
WestVirginiaUniversity
}

THE RESEARCH REPOSITORY @ WVU

Graduate Theses, Dissertations, and Problem Reports

2001

\section{Set-theoretic and algebraic properties of certain families of real functions}

Krzysztof Plotka

West Virginia University

Follow this and additional works at: https://researchrepository.wvu.edu/etd

\section{Recommended Citation}

Plotka, Krzysztof, "Set-theoretic and algebraic properties of certain families of real functions" (2001). Graduate Theses, Dissertations, and Problem Reports. 1406.

https://researchrepository.wvu.edu/etd/1406

This Dissertation is protected by copyright and/or related rights. It has been brought to you by the The Research Repository @ WVU with permission from the rights-holder(s). You are free to use this Dissertation in any way that is permitted by the copyright and related rights legislation that applies to your use. For other uses you must obtain permission from the rights-holder(s) directly, unless additional rights are indicated by a Creative Commons license in the record and/ or on the work itself. This Dissertation has been accepted for inclusion in WVU Graduate Theses, Dissertations, and Problem Reports collection by an authorized administrator of The Research Repository @ WVU.

For more information, please contact researchrepository@mail.wvu.edu. 


\title{
Set-theoretic and Algebraic Properties of Certain Families of Real Functions
}

\author{
Krzysztof Płotka \\ Dissertation submitted to the \\ Eberly College of Arts and Sciences \\ at West Virginia University \\ in partial fulfillment of the requirements for \\ the degree of \\ Doctor of Philosophy \\ in \\ Mathematics
}

Krzysztof Chris Ciesielski, Ph.D., Chair

Dening Li, Ph.D.

Magdalena Niewiadomska-Bugaj, Ph.D.

Jerzy Wojciechowski, Ph.D.

Cun-Quan Zhang, Ph.D.

Department of Mathematics

Morgantown, West Virginia 2001

Keywords: Darboux-like, Hamel, Sierpiński-Zygmund functions;

Martin's Axiom

Copyright (c) 2001 Krzysztof Płotka 


\section{ABSTRACT \\ Set-theoretic and Algebraic Properties of Certain Families of Real Functions}

\section{Krzysztof Płotka}

Given two families of real functions $\mathcal{F}_{1}$ and $\mathcal{F}_{2}$ we consider the following question: can every real function $f$ be represented as $f=f_{1}+f_{2}$, where $f_{1}$ and $f_{2}$ belong to $\mathcal{F}_{1}$ and $\mathcal{F}_{2}$, respectively? This question leads to the definition of the cardinal function Add: $\operatorname{Add}\left(\mathcal{F}_{1}, \mathcal{F}_{2}\right)$ is the smallest cardinality of a family $F$ of functions for which there is no function $g$ in $\mathcal{F}_{1}$ such that $g+F$ is contained in $\mathcal{F}_{2}$. This work is devoted entirely to the study of the function Add for different pairs of families of real functions. We focus on the classes that are related to the additive properties and generalized continuity.

Chapter 2 deals with the classes related to the generalized continuity. In particular, we show that Martin's Axiom (MA) implies Add(D,SZ) is infinite and Add(SZ,D) equals to the cardinality of the set of all real numbers. SZ and D denote the families of Sierpiński-Zygmund and Darboux functions, respectively. As a corollary we obtain that the proposition: every function from $\mathbb{R}$ into $\mathbb{R}$ can be represented as a sum of Sierpinski-Zygmund and Darboux functions is independent of ZFC axioms.

Chapter 3 is devoted entirely to the classes related to the concept of additivity. We introduce the definition of Hamel functions. We say that a real function is a Hamel function if its graph is a Hamel basis for the plane. Main result of this chapter is the theorem that every real function can be represented as the pointwise sum of two Hamel functions.

In Chapter 4 we investigate the function Add for pairs of classes such that one relates to the generalized continuity and the other to the additive properties. 


\section{ACKNOWLEDGMENTS}

I wish to express my gratitude to my advisor, Dr. K. C. Ciesielski, for his guidance, advice, and encouragement.

I would also like to thank other committee members: Dr. D. Li, Dr. M. Niewia-

domska-Bugaj, Dr. J. Wojciechowski, and C. Q. Zhang, for their help during my studies. 


\section{Contents}

1 Preliminaries 1

1.1 Introduction . . . . . . . . . . . . . . . . . . . 1

1.2 Notation, definitions, and basic facts . . . . . . . . 3

2 Classes related to generalized continuity $\quad 10$

2.1 Main result and its consequences . . . . . . . . . . . . 10

2.2 Lemmas . . . . . . . . . . . . . . . . . . . . 14

2.3 Proof of the main result $\ldots \ldots \ldots \ldots \ldots$

2.4 Sierpiński-Zygmund sets on the plane . . . . . . . . . . . 26

3 Classes related to additive properties 33

3.1 Functions with linearly independent graphs . . . . . . . . . . . . 34

3.2 Hamel functions . . . . . . . . . . . . . . . . . . . . . 37

4 Generalized continuity versus additivity 51

4.1 Introduction . . . . . . . . . . . . . . . . . 51

4.2 Relations among Darboux-like families . . . . . . . . . . 56

4.3 Relations between Darboux-like and additive or Hamel functions . . . 57 


\section{Chapter 1}

\section{Preliminaries}

\section{$1.1 \quad$ Introduction}

The classes of functions related to generalized continuity have been heavily studied in recent years. A class which generalizes some notion of continuity is called Darbuoxlike. An example of a Darbuox-like family is a class of Darboux functions. In 1875 G. Darboux [8], investigating the properties of derivatives, proved that every derivative of a real function defined on $\mathbb{R}$ has the intermediate value property. Recall that a function $f: \mathbb{R} \rightarrow \mathbb{R}$ satisfies the intermediate value property if for all real numbers $a$ and $b(a<b)$ and for every $y$ between $f(a)$ and $f(b)$ there is a real number

$c \in(a, b)$ such that $f(c)=y$. Today the class of Darboux functions is defined to be exactly the class of all functions satisfying the intermediate value property. The following example shows that continuous functions form a proper subfamily of Darboux functions. Define $f: \mathbb{R} \rightarrow \mathbb{R}$ by

$$
f(x)= \begin{cases}\sin \left(\frac{1}{x}\right) & \text { if } x \neq 0 \\ 0 & \text { otherwise. }\end{cases}
$$

There are many other families of Darbuox-like functions that we will define in the next section. 
Another example of a class which is related to the concept of continuity is the class of Sierpinski-Zygmund functions. However, it is not an example of a Darbuox-like family. A function $h: \mathbb{R} \rightarrow \mathbb{R}$ is called Sierpiński-Zygmund if every restriction of $h$ to a set of cardinality continuum is discontinuous. Hence we could say that SierpińskiZygmund functions are "anti-continuous." The existence of such a function was proved by W. Sierpiński and A. Zygmund [23]. It turns out that it is possible (under special set-theoretical assumptions) that such a pathological function can also be Darboux. On the other hand, there exists a model of set theory ZFC in which no Sierpiński-Zygmund function is Darboux. The relationship between Darboux-like families and the class of Sierpiński-Zygmund functions will be the main topic of Chapter 2.

Another notion which is also very useful in Real Analysis is additivity. This concept dates back to the early 19th century when the following functional equation was considered for the first time

$$
f(x+y)=f(x)+f(y) \text { for all } x, y \in \mathbb{R} .
$$

An obvious solution to this equation is a linear function, that is, a function defined by $f(x)=a x$ for all $x \in \mathbb{R}$, where $a$ is some constant. The first mathematician, who proved that the linear functions are the only continuous solutions, was A. L. Cauchy [2]. Because of this, the above equation is known as Cauchy's Functional Equation. For a long time the existence of a discontinuous solutions of the Cauchy equation was an open problem. This problem was solved by G. Hamel in 1905 [10] who constructed a discontinuous function which satisfies the desired equation. The key in his construction is a linear basis of $\mathbb{R}$ considered as a linear space over the rational 
numbers $\mathbb{Q}$. Such bases are called today Hamel bases. They play very important role in many constructions in Real Analysis and other areas of mathematics.

The family of all solutions of the above functional equation is called the family of additive functions. Similarly as in the case of generalized continuity, we also consider a class of functions that could be treated as "anti-additive." We say that a real function defined on $\mathbb{R}$ is a Hamel function if its graph is a Hamel basis for the plane. It is obvious that such functions cannot be additive. The class of Hamel functions and its relation to the additive functions is discussed in details in Chapter 3.

It is also of interest to compare the two concepts mentioned above: generalized continuity and additivity. The investigation of the relationship between Darboux-like classes and the additive functions is presented in Chapter 4.

\subsection{Notation, definitions, and basic facts}

The terminology and notation is standard and follows [3]. The symbols $\mathbb{R}$ and $\mathbb{Q}$ stand for the sets of all real and all rational numbers, respectively. A basis of $\mathbb{R}^{n}$ as a linear space over $\mathbb{Q}$ is called a Hamel basis. For $Y \subset \mathbb{R}^{n}$, the $\operatorname{symbol}_{\operatorname{Lin}}(Y)$ stands for the smallest linear subspace of $\mathbb{R}^{n}$ over $\mathbb{Q}$ that contains $Y$.

The cardinality of a set $X$ we denote by $|X|$. In particular, $|\mathbb{R}|$ is denoted by c. For the cardinal number $\kappa$ we write $[X]^{\kappa}$ to denote the family of all subsets $Y$ of $X$ with $|Y|=\kappa$. In particular, $[X]^{1}$ stands for the family of all singletons in $X$. Similarly we define $[X]^{<\kappa}$. If $\gamma$ is also a cardinal number then $\kappa^{<\gamma}$ denotes the cardinality of the set $[\kappa]^{<\gamma}$. The symbol $\mathrm{cf}(\kappa)$ stands for the cofinality of $\kappa$. We say that $\kappa$ is regular provided that $\operatorname{cf}(\kappa)=\kappa$. MA and $\mathrm{CH}$ are used to denote Martin's Axiom and Continuum Hypothesis, respectively. 
$\mathcal{B}$ and $\mathcal{M}$ stand for the families of all Borel and all meager subsets of $\mathbb{R}$, respectively. We say that a set $B \subseteq \mathbb{R}$ is a Bernstein set if both $B$ and $\mathbb{R} \backslash B$ intersect every perfect set. For a cardinal number $\kappa$, a set $A \subseteq \mathbb{R}$ is called $\kappa$-dense if $|A \cap I| \geq \kappa$ for every non-trivial interval $I$. For any set $P \subseteq X \times Y$, we denote its $x$-projection by $\operatorname{dom}(P)$. That is $\operatorname{dom}(P)=\{x \in X:\langle x, y\rangle \in P\}$.

We consider only real-valued functions defined on subsets of $\mathbb{R}^{n}$. No distinction is made between a function and its graph. For any two partial real functions $f$ and $g$ we write $f+g, f-g$ for the sum and difference functions defined on $\operatorname{dom}(f) \cap \operatorname{dom}(g)$. The class of all functions from a set $X$ into a set $Y$ is denoted by $Y^{X}$. We write $f \mid A$ for the restriction of $f \in Y^{X}$ to the set $A \subseteq X$. The image and preimage of a set $B$ under the function $f$ are denoted by $f[B]$ and $f^{-1}[B]$, respectively. For $C \subseteq \mathbb{R}^{n}$, its characteristic function is denoted by $\chi_{C}$. If $f, g \in Y^{X}$, then $[f \neq g]$ denotes the set $\{x \in X: f(x)=g(x)\}$. In a similar way we define. For any function $g \in \mathbb{R}^{X}$ and any family of functions $F \subseteq \mathbb{R}^{X}$ we define $g+F=\{g+f: f \in F\}$.

The cardinal function $\mathrm{A}(\mathcal{F})$, for $\mathcal{F} \subseteq \mathbb{R}^{X}$, is defined as the smallest cardinality of a family $F \subseteq \mathbb{R}^{X}$ for which there is no $g \in \mathbb{R}^{X}$ such that $g+F \subseteq \mathcal{F}$. That is

$$
\mathrm{A}(\mathcal{F})=\min \left\{|F|: F \subseteq \mathbb{R}^{X} \& \neg \exists g \in \mathbb{R}^{X} g+F \subseteq \mathcal{F}\right\} \cup\left\{\left(\left|\mathbb{R}^{X}\right|\right)^{+}\right\} .
$$

For example, if Const is the family of all constant functions from $\mathbb{R}$ to $\mathbb{R}$ then $\mathrm{A}($ Const $)=2$. To see that $\mathrm{A}($ Const $) \geq 2$ choose any function $f \in \mathbb{R}^{\mathbb{R}}$. Notice that $f+(-f) \in$ Const, so $\mathrm{A}$ (Const) $\geq 2$. To prove the opposite inequality let us define $f_{1}$ and $f_{2}$ to be characteristic functions of $\{1\}$ and $\{2\}$, respectively. Now, if $g+f_{1} \in$ Const for some $g \in \mathbb{R}^{\mathbb{R}}$ then $g+f_{2}=\left(g+f_{1}\right)+\left(f_{2}-f_{1}\right) \notin$ Const.

The function A was investigated for many different classes of real functions, see 
e.g. [6], [7], [18]. In this work we generalize the function $\mathrm{A}$ by imposing some restrictions on the function $g$. Thus for $\mathcal{F}_{1}, \mathcal{F}_{2} \subseteq \mathbb{R}^{X}$ we define

$$
\operatorname{Add}\left(\mathcal{F}_{1}, \mathcal{F}_{2}\right)=\min \left\{|F|: F \subseteq \mathbb{R}^{X} \& \neg \exists g \in \mathcal{F}_{1} g+F \subseteq \mathcal{F}_{2}\right\} \cup\left\{\left(\left|\mathbb{R}^{X}\right|\right)^{+}\right\}
$$

Observe that $\mathrm{A}(\mathcal{F})=\operatorname{Add}\left(\mathbb{R}^{X}, \mathcal{F}\right)$ for any set $X$, so the function Add is indeed a generalization of the function A. Notice also the following properties of the Add function.

Proposition 1.2.1 Let $\mathcal{F}_{1} \subseteq \mathcal{F}_{2} \subseteq \mathbb{R}^{X}$ and $\mathcal{F} \subseteq \mathbb{R}^{X}$.

(1) $\operatorname{Add}\left(\mathcal{F}_{1}, \mathcal{F}\right) \leq \operatorname{Add}\left(\mathcal{F}_{2}, \mathcal{F}\right)$.

(2) $\operatorname{Add}\left(\mathcal{F}, \mathcal{F}_{1}\right) \leq \operatorname{Add}\left(\mathcal{F}, \mathcal{F}_{2}\right)$.

(3) $\operatorname{Add}\left(\mathcal{F}_{1}, \mathcal{F}_{2}\right) \geq 2$ if and only if $\mathbb{R}^{X}=\mathcal{F}_{2}-\mathcal{F}_{1}$.

(4) If $\operatorname{Add}\left(\mathcal{F}_{1}, \mathcal{F}_{2}\right) \geq 2$ then $\mathcal{F}_{1} \cap \mathcal{F}_{2} \neq \emptyset$.

(5) $\mathrm{A}(\mathcal{F})=\operatorname{Add}(\mathcal{F}, \mathcal{F})+1$. In particular, if $\mathrm{A}(\mathcal{F}) \geq \omega$ then $\operatorname{Add}(\mathcal{F}, \mathcal{F})=\mathrm{A}(\mathcal{F}) .{ }^{1}$

Proof. (1) Let $G \subseteq \mathbb{R}^{X}$ be such that $|G|<\operatorname{Add}\left(\mathcal{F}_{1}, \mathcal{F}\right)$. From the definition of Add we get that there exists a $g \in \mathcal{F}_{1}$ with the property that $g+G \in \mathcal{F}$. Since $g \in \mathcal{F}_{1} \subseteq \mathcal{F}_{2}$ we obtain that $\operatorname{Add}\left(\mathcal{F}_{1}, \mathcal{F}\right) \leq \operatorname{Add}\left(\mathcal{F}_{2}, \mathcal{F}\right)$.

(2) The proof of (2) is very similar to the proof of (1).

(3) Assume that $\operatorname{Add}\left(\mathcal{F}_{1}, \mathcal{F}_{2}\right) \geq 2$. Based on the definition of Add, this is equivalent to

$$
\forall f \in \mathbb{R}^{X} \exists f_{1} \in \mathcal{F}_{1} \quad \exists f_{2} \in \mathcal{F}_{2} \text { such that } f_{1}+f=f_{2} .
$$

\footnotetext{
${ }^{1}$ Very similar observation, in a little bit different context, was obtained independently by Francis Jordan [12, Proposition 1.3].
} 
From this we obtain that $f=f_{1}-f_{2}$. Thus, $f \in \mathcal{F}_{2}-\mathcal{F}_{1}$ for all $f \in \mathbb{R}^{X}$. Consequently, $\mathbb{R}^{X}=\mathcal{F}_{2}-\mathcal{F}_{1}$.

Notice that the above argument can be reserved. Thus, we proved the equivalency.

(4) Part (3) implies that if $\operatorname{Add}\left(\mathcal{F}_{1}, \mathcal{F}_{2}\right) \geq 2$ then $\mathbf{0} \in \mathcal{F}_{2}-\mathcal{F}_{1}$, where $\mathbf{0}: X \rightarrow \mathbb{R}$ is a function identically equal to zero. Hence there are $f_{1} \in \mathcal{F}_{1}$ and $f_{2} \in \mathcal{F}_{2}$ such that $\mathbf{0}=f_{1}-f_{2}$. So $f_{1}=f_{2}$ and consequently $\mathcal{F}_{1} \cap \mathcal{F}_{2} \neq \emptyset$.

(5) By (1) $\operatorname{Add}(\mathcal{F}, \mathcal{F}) \leq \operatorname{Add}\left(\mathbb{R}^{X}, \mathcal{F}\right)=\mathrm{A}(\mathcal{F})$. On the other hand, observe that $\mathrm{A}(\mathcal{F}) \leq \operatorname{Add}(\mathcal{F}, \mathcal{F})+1$. To see the above let $F \subseteq \mathbb{R}^{\mathbb{R}}$ be such that $|F|=\operatorname{Add}(\mathcal{F}, \mathcal{F})$ and

$$
\neg \exists g \in \mathcal{F} g+F \subseteq \mathcal{F} .
$$

Then we have

$$
\neg \exists g \in \mathbb{R}^{\mathbb{R}} g+(F \cup\{\mathbf{0}\}) \subseteq \mathcal{F} .
$$

So the conclusion is obvious in the case $\mathrm{A}(\mathcal{F}) \geq \omega$. Therefore we will concentrate on the case $\mathrm{A}(\mathcal{F})=k$ for some $k \in \omega$. Recall that the function $\mathrm{A}$ is bounded from the bottom by 1 , thus $k \geq 1$. From the previous argument we conclude that $\operatorname{Add}(\mathcal{F}, \mathcal{F}) \geq k-1$. So we only need to justify that $\operatorname{Add}(\mathcal{F}, \mathcal{F}) \leq k-1$.

Let $\left\{f_{1}, \ldots, f_{k}\right\}$ be a family witnessing $\mathrm{A}(\mathcal{F})=k$. Then the set $\left\{f_{i}-f_{k}\right\}_{i=1}^{k-1}$ witnesses $\operatorname{Add}(\mathcal{F}, \mathcal{F}) \leq k-1$. Indeed, assume by contradiction, that we can find a function $f \in \mathcal{F}$ such that $\left(f_{i}-f_{k}\right)+f \in \mathcal{F}$ for every $i=1, \ldots, k-1$. Then the function $f-f_{k}$ shifts the set $\left\{f_{1}, \ldots, f_{k}\right\}$ into $\mathcal{F}$, a contradiction.

The following is a list of the definitions of the different types of functions that we mentioned in the previous section. These classes are main focus of this work.

For $X \subseteq \mathbb{R}^{n}$ a function $f: X \rightarrow \mathbb{R}$ is: 
- additive if $f(x+y)=f(x)+f(y)$ for all $x, y \in X$ such that $x+y \in X$;

- almost continuous (in sense of Stallings) if each open subset of $X \times \mathbb{R}$ containing the graph of $f$ contains also the graph of a continuous function from $X$ to $\mathbb{R}$;

- connectivity if the graph of $f \mid Z$ is connected in $Z \times \mathbb{R}$ for any connected subset $Z$ of $X$;

- Darboux if $f[K]$ is a connected subset of $\mathbb{R}$ (i.e., an interval) for every connected subset $K$ of $X$;

- an extendability function provided there exists a connectivity function $F$ from $X \times[0,1]$ into $\mathbb{R}$ such that $f(x)=F(x, 0)$ for every $x \in X$;

- a Hamel function provided that the graph of $f$ is a Hamel basis for $\mathbb{R}^{n+1}$;

- peripherally continuous if for every $x \in X$ and for all pairs of open sets $U$ and $V$ containing $x$ and $f(x)$, respectively, there exists an open subset $W$ of $U$ such that $x \in W$ and $f[\operatorname{bd}(W)] \subset V$;

- Sierpiński-Zygmund if for every set $Y \subseteq X$ of cardinality continuum $\mathfrak{c}, f \mid Y$ is discontinuous.

The classes of functions defined above are denoted by $\operatorname{AD}(X), \operatorname{AC}(X), \operatorname{Conn}(X)$, $\mathrm{D}(X), \operatorname{Ext}(X), \mathrm{HF}(X), \mathrm{PC}(X)$, and $\mathrm{SZ}(X)$, respectively. The family of all continuous functions from $X$ into $\mathbb{R}$ is denoted by $\mathrm{C}(X)$. We drop the index $X$ in the case $X=\mathbb{R}$. To simplify notation, we introduce the symbol $\mathrm{SZ}_{\mathrm{part}}$ to denote $\bigcup_{X \subseteq \mathbb{R}} \mathrm{SZ}(X)$.

Recall that a function $f: \mathbb{R}^{n} \rightarrow \mathbb{R}$ is almost continuous if and only if it intersects every blocking set, i.e., a closed set $K \subseteq \mathbb{R}^{n+1}$ which meets every continuous function 
from $C\left(\mathbb{R}^{n}\right)$ and is disjoint with at least one function from $\mathbb{R}^{\mathbb{R}^{n}}$. The domain of every blocking set contains a non-degenerate connected set. (See [14].) It is also well-known that each continuous partial function can be extended to a continuous function defined on some $G_{\delta}$ set. (See [17].) Thus if $|[f=g]|<\mathfrak{c}$ for each continuous partial function $g$ defined on some $G_{\delta}$-set then $f$ is Sierpiński-Zygmund. Recall also that each additive function $f \in \mathrm{AD}$ is linear over $\mathbb{Q}$, i.e., for all $p, q \in \mathbb{Q}$ and $x, y \in \mathbb{R}$ we have $f(p x+q y)=p f(x)+q f(y)$.

The above classes are related in the following way (arrows $\longrightarrow$ indicate proper inclusions.) (See [4] or [9].)

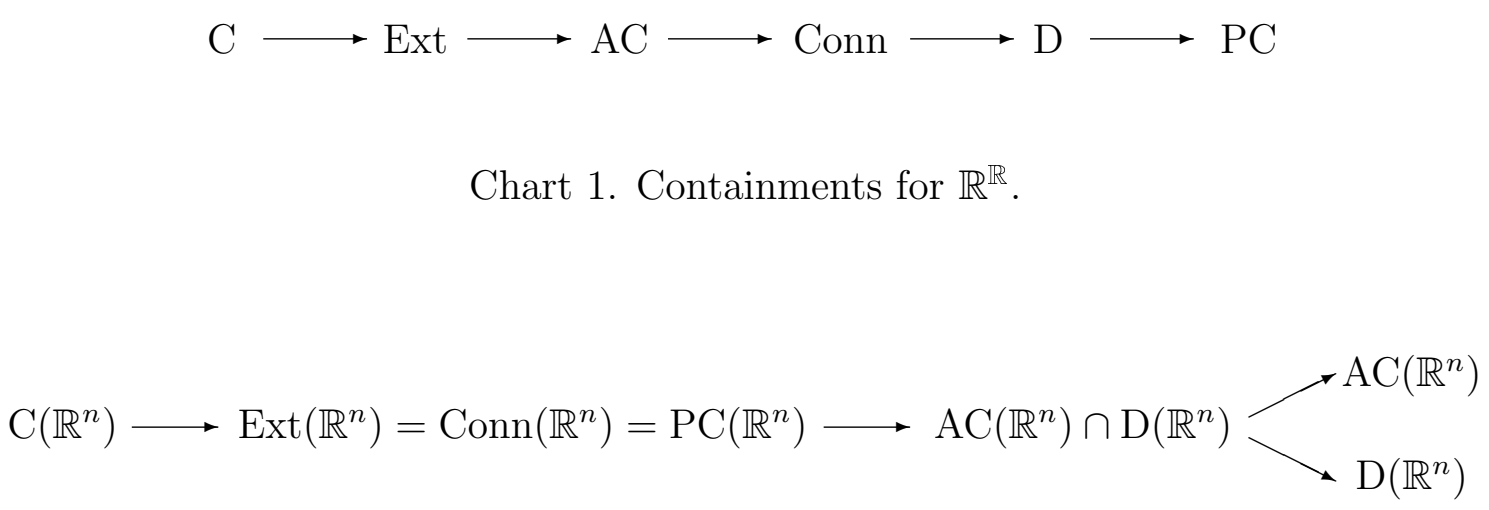

Chart 2. Containments for $\mathbb{R}^{\mathbb{R}^{n}}$ when $n \geq 2$.

The class of Sierpiński-Zygmund functions is independent of all the classes included in the above chart in the following sense. There is no inclusion between SZ and AC, Conn, D, or PC. SZ is disjoint with $\mathrm{C}$ and Ext. (See also comment below Corollary 2.1.4.) $\mathrm{SZ}\left(\mathbb{R}^{n}\right)$ is disjoint with $\mathrm{D}\left(\mathbb{R}^{n}\right)$ and $\mathrm{AC}\left(\mathbb{R}^{n}\right)$ for $n \geq 2$. (See Remarks 2.1.6 and 2.1.7.) 
The class of additive functions $\operatorname{AD}\left(\mathbb{R}^{n}\right)$ intersects each of the other classes (the existence of a function in $\mathrm{AD} \cap \mathrm{SZ}$ follows from Theorem 4.3.1 (iv) and Proposition 1.2.1 (4).) However, it is not contained in any of them except the family $\mathrm{PC}\left(\mathbb{R}^{n}\right)$ in the case $n=1$. Then we have $\mathrm{AD} \subseteq \mathrm{PC}$.

For the relationship between Hamel functions and the other families see Section 4.3 .

Now let us comment on $\mathrm{A}(\mathcal{F})$ for $\mathcal{F} \in\{$ Ext, $\mathrm{AC}$, Conn, D, PC, SZ $\}$. The following can be proved in ZFC:

$$
\begin{gathered}
\mathfrak{c}^{+}=\mathrm{A}(\mathrm{Ext}) \leq \mathrm{A}(\mathrm{AC})=\mathrm{A}(\mathrm{Conn})=\mathrm{A}(\mathrm{D}) \leq \mathrm{A}(\mathrm{PC})=2^{\mathfrak{c}}, \\
\mathfrak{c}^{+} \leq \mathrm{A}(\mathrm{SZ}) \leq 2^{\mathfrak{c}} .
\end{gathered}
$$

For more details see [5], [6], [7], and [18]. The number A(HF) will be investigated in Chapter 3.

Before we finish this section it is useful to define the class of countably continuous functions. It will not be in our focus but it will be used many times in the proofs. We say that a function $f: X \rightarrow \mathbb{R}\left(X \subseteq \mathbb{R}^{n}\right)$ is countably continuous if it can be represented as a union of countably many continuous partial functions. Shortly we write $f \in \mathrm{CC}(X)$ or, in the case $X=\mathbb{R}, f \in \mathrm{CC}$. We also introduce the symbol $\mathrm{CC}_{\text {part }}$ to denote $\bigcup_{X \subseteq \mathbb{R}} \mathrm{CC}(X)$. 


\section{Chapter 2}

\section{Classes related to generalized continuity}

The main focus of this chapter is the relationship between the Darboux-like families (from Charts 1 and 2) and the class of Sierpiński-Zygmund functions. Most of the following material (excluding the last section) is based on my paper [20]. Section 2.4 includes results from [22].

In the next section we present the main result and discuss its consequences. In Section 2.2 we state and prove two auxiliary lemmas that are also of interest on their own. Section 2.3 is devoted to the proof of the main result. The proof is based on the above lemmas. Finally, in Section 2.4 we generalize the concept of SierpińskiZygmund functions by defining Sierpiński-Zygmund sets. Then we state and prove some properties of these sets.

\subsection{Main result and its consequences}

Let us start with considering the following problem.

Given two families $\mathcal{F}_{1}, \mathcal{F}_{2} \subseteq \mathbb{R}^{\mathbb{R}}$ of real functions, can every function $f \in \mathbb{R}^{\mathbb{R}}$ 
be represented as $f=f_{1}+f_{2}$, where $f_{i} \in \mathcal{F}_{i}$ for $i=1,2$ ? In other words, does $\mathcal{F}_{1}+\mathcal{F}_{2}=\left\{f_{1}+f_{2}: f_{i} \in \mathcal{F}_{i}, i=1,2\right\}$ equal to $\mathbb{R}^{\mathbb{R}}$ ?

Obviously, the answer to the above question depends on the properties of these families. For example, if $\mathcal{F}_{1}$ and $\mathcal{F}_{2}$ are too "good" in some sense (i.e., in terms of continuity) then the answer will be negative. On the other hand, we may be interested what happens if one of $\mathcal{F}_{1}, \mathcal{F}_{2}$ is "good" and the other one is "bad" in the same sense? In particular, can we prove that every real function is a sum of two functions such that one is continuous and the other one "extremely discontinuous", say SierpińskiZygmund? It is easy to see that if $f_{1} \in \mathrm{C}$ and $f_{2} \in \mathrm{SZ}$ then $f_{1}+f_{2} \in \mathrm{SZ} \neq \mathbb{R}^{\mathbb{R}}$. Thus, the last question has a negative answer. Can we weaken somehow the concept of continuity so that we get an affirmative answer? What will happen if the continuous functions are replaced by the almost continuous functions? The answers to this and many other related questions will be implied by our main result, that follows

\section{Theorem 2.1.1.}

(1) $(\mathrm{MA}) \operatorname{Add}(\mathrm{D}, \mathrm{SZ}) \geq \operatorname{Add}(\mathrm{AC}, \mathrm{SZ}) \geq \omega$.

(2) $(\mathrm{MA}) \operatorname{Add}(\mathrm{SZ}, \mathrm{AC})=\operatorname{Add}(\mathrm{SZ}, \mathrm{D})=\mathfrak{c}$.

(3) If the theory "ZFC $+\exists$ measurable cardinal" is consistent then so is "ZFC + $\operatorname{Add}(\mathrm{AC}, \mathrm{SZ})>\mathfrak{c}>\omega_{1} . "$

(4) $\operatorname{Add}(\mathrm{PC}, \mathrm{SZ})=\mathrm{A}(\mathrm{SZ})$ and $\operatorname{Add}(\mathrm{SZ}, \mathrm{PC})=2^{\mathfrak{c}}$.

The following remains an open problem. (See Fact 2.2.4.) 
Problem 2.1.2 Does the equality $\mathrm{Add}(\mathrm{AC}, \mathrm{SZ})=\omega$ hold in "ZFC + MA" (or in "ZFC + $\mathrm{CH} " ?)$

Let us make here some comments about the theorem. Parts (1) and (3) give only lower bound for $\operatorname{Add}(\mathrm{AC}, \mathrm{SZ})$. So one may wonder whether it is possible to give in ZFC any non-trivial upper bound for that number. However, in the model used to prove (3) it is possible to have $\mathfrak{c}^{+}=2^{\mathfrak{c}}$, so it cannot be proved in ZFC that $\operatorname{Add}(\mathrm{AC}, \mathrm{SZ})<2^{\mathfrak{c}}$. But it is unknown whether $\operatorname{Add}(\mathrm{AC}, \mathrm{SZ}) \leq \mathfrak{c}^{+}$holds in ZFC. The next comment is about symmetry of Add. It is consistent that $\mathrm{A}(\mathrm{SZ})<2^{\mathfrak{c}}$. (See [6].) Hence part (4) implies that Add is not symmetric in general.

Next we give some corollaries of the main result. To state the first one, note that $-\mathrm{SZ}=\{-f: f \in \mathrm{SZ}\}=\mathrm{SZ}$. This observation, Proposition 1.2.1 and part (2) of Theorem 2.1.1 immediately imply the following corollary.

Corollary 2.1.3 (MA) Every function $f: \mathbb{R} \rightarrow \mathbb{R}$ can be represented as a sum of almost continuous and Sierpiński-Zygmund functions.

Let us mention that the corollary, so also parts (1) and (2) of Theorem 2.1.1, cannot be proved in ZFC alone (i.e., without any additional assumptions.) Indeed, if $\mathbb{R}^{\mathbb{R}}=\mathrm{AC}+\mathrm{SZ}$ then, by Proposition 1.2.1 (4), there exists an almost continuous function which is also Sierpiński-Zygmund. An example of a model with no Darboux (so also almost continuous) Sierpiński-Zygmund function is given in [1]. Hence we can state

Corollary 2.1.4 The equalities $\mathbb{R}^{\mathbb{R}}=\mathrm{AC}+\mathrm{SZ}$ and $\mathbb{R}^{\mathbb{R}}=\mathrm{D}+\mathrm{SZ}$ are independent of ZFC. 
One may ask whether Corollary 2.1.3 can be improved by replacing the family AC of almost continuous functions by the family Ext of extendable functions. However, it cannot be done. The reason is that every extendable function is continuous on some perfect set. (See [4].) The above observation implies

Fact 2.1.5 $\operatorname{Add}($ Ext, SZ $)=\operatorname{Add}(S Z, E x t)=1$.

One may also try to generalize Corollary 2.1.3 for all functions from $\mathbb{R}^{n}$ into $\mathbb{R}$. However, in the case $n \geq 2$ it can be proved in ZFC that there is no almost continuous function which is also Sierpiński-Zygmund. We have the following remark.

Remark 2.1.6 Let $n \geq 2$. Then $\mathrm{AC}\left(\mathbb{R}^{n}\right) \cap \mathrm{SZ}\left(\mathbb{R}^{n}\right)=\emptyset$ and

$$
\operatorname{Add}\left(\operatorname{AC}\left(\mathbb{R}^{n}\right), \operatorname{SZ}\left(\mathbb{R}^{n}\right)\right)=\operatorname{Add}\left(\operatorname{SZ}\left(\mathbb{R}^{n}\right), \operatorname{AC}\left(\mathbb{R}^{n}\right)\right)=1
$$

Proof. For every $n \geq 2$ if $f \in \mathrm{AC}\left(\mathbb{R}^{n}\right) \cap \mathrm{SZ}\left(\mathbb{R}^{n}\right)$ then $f \mid \mathbb{R}^{2} \in \mathrm{AC}\left(\mathbb{R}^{2}\right) \cap \mathrm{SZ}\left(\mathbb{R}^{2}\right)$. (See [18].) Hence it is enough to prove the remark for $n=2$. We construct the family $\left\{B_{y}: y \in \mathbb{R}\right\}$ of $\mathfrak{c}$-many blocking sets in $\mathbb{R}^{3}$ with pairwise disjoint $x y$-projections and whose union is the graph of a continuous function. Let $B_{y}=\{\langle x, y, \tan (x)\rangle: x \in$ $\left.\left(\frac{-\pi}{2}, \frac{\pi}{2}\right)\right\}$ for $y \in \mathbb{R}$. Every almost continuous function from $\mathbb{R}^{2}$ to $\mathbb{R}$ must intersect all sets $B_{y}$. Thus it cannot be of Sierpiński-Zygmund type, since it agrees with the function $F(x, y)=\tan (x)$ on a set of cardinality of continuum.

The second part of the conclusion follows from Proposition 1.2.1 (4).

Let us finish by making a comment about $\operatorname{Add}\left(\mathrm{D}\left(\mathbb{R}^{n}\right), \mathrm{SZ}\left(\mathbb{R}^{n}\right)\right)$. It is easy to see that $\mathrm{SZ}\left(\mathbb{R}^{n}\right) \cap \mathrm{D}\left(\mathbb{R}^{n}\right)=\emptyset$ because for each non-constant Darboux function $f: \mathbb{R}^{n} \rightarrow \mathbb{R}$ there exists a real number $y$ such that $f^{-1}(y)$ disconnects $\mathbb{R}^{n}$. Based on this we obtain Remark 2.1.7 $\operatorname{Add}\left(\mathrm{D}\left(\mathbb{R}^{n}\right), \mathrm{SZ}\left(\mathbb{R}^{n}\right)\right)=\operatorname{Add}\left(\mathrm{SZ}\left(\mathbb{R}^{n}\right), \mathrm{D}\left(\mathbb{R}^{n}\right)\right)=1$ for $n \geq 2$. 


\section{$2.2 \quad$ Lemmas}

This section consists of two auxiliary lemmas. To state the lemmas we need the following definitions. For $X \subseteq \mathbb{R}$ by $\mathrm{C}^{<\mathfrak{c}}(X)$ we denote the family of all functions $f: X \rightarrow \mathbb{R}$ which can be represented as a union of less than $\mathfrak{c}$-many partial continuous functions. To simplify notation we write $\mathrm{C}^{<\mathfrak{c}}$ and $\mathrm{C}_{\text {part }}^{<\mathfrak{c}}$ for $\mathrm{C}^{<\mathfrak{c}}(\mathbb{R})$ and $\bigcup_{X \subseteq \mathbb{R}} \mathrm{C}^{<\mathfrak{c}}(X)$, respectively. Observe that under the assumption of regularity of $\mathfrak{c}$ (so also under MA) $\mathrm{SZ}(X)+\mathrm{C}^{<\mathfrak{c}}(X)=\mathrm{SZ}(X)$ and $\mathrm{SZ}(Y) \cap \mathrm{C}^{<\mathfrak{c}}(Y)=\emptyset$ for any $X, Y \subseteq \mathbb{R}$ with $|Y|=\mathfrak{c}$.

The same assumption about $\mathfrak{c}$ implies also that the union of any family $F \subseteq \mathrm{C}_{\text {part }}^{<\mathfrak{c}}$ of cardinality less than $\mathfrak{c}$ contains a function from $\mathrm{C}^{<\mathfrak{c}}\left(\bigcup_{f \in F} \operatorname{dom}(f)\right)$.

Now we introduce the next definition. Let $A \subseteq \mathbb{R}$ be everywhere of second category, that is $A \cap I$ is of second category for every nontrivial interval $I$. We define $\mathcal{F}_{A}$ as a family of all $F \subseteq \mathbb{R}^{\mathbb{R}}$ whose union $\bigcup F$ contains no function from $\mathrm{C}^{<\mathfrak{r}}(A \cap B)$ for any non-meager Borel set $B$. That is

$$
\mathcal{F}_{A}=\left\{F \subseteq \mathbb{R}^{\mathbb{R}}: \forall B \in(\mathcal{B} \backslash \mathcal{M}) \forall f \in \mathrm{C}^{<\mathfrak{c}}(A \cap B) f \nsubseteq \bigcup F\right\}
$$

Lemma 2.2.1 (MA) Let $F \in \mathcal{F}_{A}$ be a family such that $|F|<\mathrm{A}(\mathrm{SZ})$. There exists a $g \in \mathrm{SZ}(A)$ such that $g+F \subseteq \mathrm{SZ}(A)$ and for every blocking set $B \subseteq \mathbb{R}^{2}$ there is a non-empty open interval $I_{B} \subseteq \operatorname{dom}(B)$ with the property that $\operatorname{dom}(B \cap g)$ is dense in $I_{B}$. In particular, every extension $\bar{g}: \mathbb{R} \rightarrow \mathbb{R}$ of $g$ is almost continuous.

Proof. Let $\left\langle f_{\alpha}: \alpha<\mathfrak{c}\right\rangle$ be a sequence of all continuous functions defined on $G_{\delta}$ subsets of $\mathbb{R}$.

(1) First we construct a partial real function $g^{\prime} \in \mathrm{SZ}_{\text {part }}$ with $\operatorname{dom}\left(g^{\prime}\right) \subseteq A$ and having the properties as in the lemma. We do this by transfinite induction. We 
construct a sequence $\left\langle g_{\xi}: \xi<\mathfrak{c}\right\rangle$ of partial real functions satisfying the following conditions for every $\alpha<\mathfrak{c}$ :

(a) $D_{\alpha}=\operatorname{dom}\left(g_{\alpha}\right)$ is countable;

(b) $g_{\alpha}$ is a dense subset of $\left(f_{\alpha} \mid A\right) \backslash \bigcup_{\xi<\alpha}\left(f_{\xi} \cup\left(D_{\xi} \times \mathbb{R}\right) \cup \bigcup\left(f_{\xi}-F\right)\right)$.

Notice that $D_{\alpha} \cap D_{\beta}=\emptyset$ and $D_{\alpha} \subseteq A$ for $\alpha<\beta<\mathfrak{c}$. Now we define $g^{\prime}=\bigcup_{\xi<\mathfrak{c}} g_{\xi}$. We will show that $g^{\prime}$ has the required properties.

(i) $g^{\prime}, g^{\prime}+f \in \mathrm{SZ}_{\text {part }}$ for every $f \in F$.

Let $\xi<\mathfrak{c}$. We see from the condition (b) that $\left[g^{\prime}=f_{\xi}\right],\left[\left(g^{\prime}+f\right)=f_{\xi}\right] \subseteq \bigcup_{\alpha \leq \xi} D_{\alpha}$. Hence $\left|\left[g^{\prime}=f_{\xi}\right]\right|,\left|\left[\left(g^{\prime}+f\right)=f_{\xi}\right]\right| \leq \xi \omega<\mathfrak{c}$.

(ii) For every blocking set $B \subseteq \mathbb{R}^{2}$ there is a non-empty open interval $I_{B} \subseteq \operatorname{dom}(B)$ with the property that $\operatorname{dom}(B \cap g)$ is dense in $I_{B}$.

$B$ contains a continuous function $q$ defined on a Borel set of second category. (See [15].) Let $\alpha_{B}$ be the smallest ordinal number such that $f_{\alpha_{B}}$ agrees with $q$ on a set residual in some interval $J \subseteq \operatorname{dom}(B)$. B is closed and therefore $f_{\alpha_{B}} \mid J \subseteq B$. From the definition of $\alpha_{B}$ and MA we see that $\bigcup_{\xi<\alpha_{B}}\left[f_{\xi}=q\right]$ is of first category as the union of less than $\mathfrak{c}$-many sets of first category. Recall that $F \in \mathcal{F}_{A}$. This implies that $(I \cap A) \backslash \bigcup_{\xi<\alpha_{B}} \bigcup_{f \in F}\left[\left(f_{\xi}-f\right)=q\right]$ is of second category for every nontrivial interval $I$. The above holds because otherwise we would have that $(K \cap A) \subseteq$ $\bigcup_{\xi<\alpha_{B}} \bigcup_{f \in F}\left[\left(f_{\xi}-f\right)=q\right]$ for some $K \in \mathcal{B} \backslash \mathcal{M}$. Then for every $x \in(K \cap A)$ there are $\xi<\alpha_{B}$ and $f \in F$ such that $f_{\xi}(x)-f(x)=q(x)$. Define $h:(K \cap A) \rightarrow \mathbb{R}$ by $h(x)=f_{\xi}(x)-q(x)=f(x)$. It is easy to see that $h$ is a subset of both $\bigcup_{\xi<\alpha_{B}}\left(f_{\xi}-q\right)$ 
and $\bigcup F$. In particular, it implies that $h \in \mathrm{C}^{<\mathfrak{r}}(K \cap A)$ which contradicts the assumption that $F \in \mathcal{F}_{A}$.

Hence $(J \cap A) \backslash \bigcup_{\xi<\alpha_{B}}\left(\bigcup_{f \in F}\left[\left(f_{\xi}-f\right)=q\right] \cup\left[f_{\xi}=q\right] \cup D_{\xi}\right)$ is of second category. Therefore $D_{\alpha_{B}}$ is dense in some non-empty open interval $I_{B} \subseteq J$. This implies that $\operatorname{dom}\left(g_{\alpha_{B}} \cap B\right)$ is dense in $I_{B}\left(g_{\alpha_{B}}\right.$ and $f_{\alpha_{B}}$ coincide on $D_{\alpha_{B}} \cap J$.) Since $g^{\prime} \cap B \supseteq g_{\alpha_{B}} \cap B$, we also have that $\operatorname{dom}\left(g^{\prime} \cap B\right)$ is dense in $I_{B}$.

(2) Let $g^{\prime \prime}: A \backslash \operatorname{dom}\left(g^{\prime}\right) \rightarrow \mathbb{R}$ be a Sierpiński-Zygmund function such that $g^{\prime \prime}+F \subseteq$ $\mathrm{SZ}_{\text {part }}$. Such a function exists because $|F|<\mathrm{A}(\mathrm{SZ})$. We define $g=g^{\prime} \cup g^{\prime \prime}$. We see that $g \in \mathrm{SZ}(A)$, any extension of $g$ onto $\mathbb{R}$ is in $\mathrm{AC}$, and $g+F \subseteq \mathrm{SZ}(A)$.

Lemma 2.2.2 (MA) Let $\left\{f_{i}\right\}_{1}^{n} \subseteq \mathbb{R}^{\mathbb{R}}, n=1,2, \ldots$ There exists $\left\{f_{i}^{\prime}\right\}_{1}^{n} \in \mathcal{F}_{A}$ such that $f_{i} \mid A_{i} \in \mathrm{C}^{<\mathfrak{r}}\left(A_{i}\right)$, where $A_{i}=\left[f_{i} \neq f_{i}^{\prime}\right]$.

Proof. The proof is by induction on number $n$ of functions.

Assume that the lemma is true for every $\left\{g_{i}\right\}_{1}^{n-1} \subseteq \mathbb{R}^{\mathbb{R}}, n \geq 1$. Let us fix $\left\{f_{i}\right\}_{1}^{n} \subseteq$ $\mathbb{R}^{\mathbb{R}}$. We will construct a family $\left\{f_{i}^{\prime}\right\}_{1}^{n} \in \mathcal{F}_{A}$ such that $f_{i} \mid\left[f_{i} \neq f_{i}^{\prime}\right] \in \mathrm{C}^{<\mathfrak{c}}\left(\left[f_{i} \neq f_{i}^{\prime}\right]\right)$ for all $i \leq n$.

We start with showing that the following claim holds for all $f, h, h^{\prime} \in \mathbb{R}^{\mathbb{R}}$.

$$
\text { If } f \mid[f \neq h] \in \mathrm{C}_{\text {part }}^{<\mathfrak{c}} \text { and } h \mid\left[h \neq h^{\prime}\right] \in \mathrm{C}_{\text {part }}^{<\mathfrak{c}} \text { then } f \mid\left[f \neq h^{\prime}\right] \in \mathrm{C}_{\text {part }}^{<\mathfrak{c}} .
$$

This is so because we have that $\left[f \neq h^{\prime}\right] \subseteq[f \neq h] \cup\left[h \neq h^{\prime}\right]$ and consequently

$$
\begin{gathered}
f\left|\left[f \neq h^{\prime}\right] \subseteq f\right|\left([f \neq h] \cup\left[h \neq h^{\prime}\right]\right)=f|[f \neq h] \cup f|\left(\left[h \neq h^{\prime}\right] \backslash[f \neq h]\right) \subseteq \\
\subseteq f|[f \neq h] \cup h|\left[h \neq h^{\prime}\right] .
\end{gathered}
$$

This completes the proof of the claim. 
Now observe that, by the inductive assumption, there exists $\left\{h_{i}\right\}_{2}^{n} \in \mathcal{F}_{A}$ such that $f_{i} \mid\left[f_{i} \neq h_{i}\right] \in \mathrm{C}_{\text {part }}^{<\mathfrak{c}}$ for $i=2, \ldots, n$. Put $h_{1}=f_{1}$. If $\left\{h_{i}^{\prime}\right\}_{1}^{n} \in \mathcal{F}_{A}$ is such that $h_{i} \mid\left[h_{i} \neq h_{i}^{\prime}\right] \in \mathrm{C}_{\text {part }}^{<\mathrm{c}}$ for $i=1, \ldots, n$ then, based on the above claim, also $f_{i} \mid\left[f_{i} \neq h_{i}^{\prime}\right] \in \mathrm{C}_{\mathrm{part}}^{<\mathfrak{c}}$ for all $i$. So without loss of generality we may assume that $\left\{f_{i}\right\}_{2}^{n} \in \mathcal{F}_{A}$.

Next we define the family $\mathcal{B}_{f_{1}, \ldots, f_{n}}$ by

$$
\mathcal{B}_{f_{1}, \ldots, f_{n}}=\left\{A \cap B: B \in \mathcal{B} \backslash \mathcal{M} \& \exists f \in \mathrm{C}^{<\mathfrak{c}}(A \cap B) f \subseteq \bigcup f_{i}\right\}
$$

There exists a maximal element $A_{\max }$ in $\mathcal{B}_{f_{1}, \ldots, f_{n}}$ with respect to the relation $\subseteq^{*}$ defined by

$$
X_{1} \subseteq^{*} X_{2} \text {, if } X_{1} \backslash X_{2} \text { is of first category. }
$$

To prove the existence let us consider $\mathcal{S}=\left\{B \in \mathcal{B} \backslash \mathcal{M}: A \cap B \in \mathcal{B}_{f_{1}, \ldots, f_{n}}\right\}$. For every $B \in \mathcal{S}$ we define a maximal open set $U_{B}$ such that $B$ is residual in $U_{B}$. Since $\mathbb{R}$ has a countable base, there is a sequence $\left\langle B_{n} \in \mathcal{S}: n<\omega\right\rangle$ such that $\bigcup_{B \in \mathcal{S}} U_{B}=\bigcup_{n<\omega} U_{B_{n}}$. We claim that $A_{\max }=\bigcup_{n<\omega}\left(A \cap B_{n}\right)$ is the desired maximal element. First we notice that $A_{\max } \in \mathcal{B}_{f_{1}, \ldots, f_{n}}$. Now, let $A \cap B \in \mathcal{B}_{f_{1}, \ldots, f_{n}}$. From the properties of the sets $B_{n}(n<\omega)$ we get that $B \subseteq^{*} U_{B} \subseteq \bigcup_{n<\omega} U_{B_{n}} \subseteq^{*} \bigcup_{n<\omega} B_{n}$. So $A \cap B \subseteq^{*} A_{\max }$.

Now, let $f$ be the function associated with $A_{\max }$ (e.g. $f \in \mathrm{C}^{<\mathfrak{c}}\left(A_{\max }\right)$ and $f \subseteq$ $\bigcup f_{i}$ ). The function $f$ can be represented as $f=\bigcup f_{i} \mid A_{i}$, where $\bigcup_{i \leq n} A_{i}=A_{\max }$, $A_{i} \cap A_{j}=\emptyset(i \neq j)$, and $f_{i} \mid A_{i} \in \mathrm{C}^{<\mathfrak{r}}\left(A_{i}\right)$. Let us consider the following functions $f_{i}^{\prime}=f_{i} \mid\left(\mathbb{R} \backslash A_{i}\right) \cup g_{i}$, where $g_{i} \in \mathrm{SZ}\left(A_{i}\right)(i=1, \ldots, n)$. We will show that $\left\{f_{i}^{\prime}\right\}_{1}^{n}$ is the required family, that is $\left\{f_{i}^{\prime}\right\}_{1}^{n} \in \mathcal{F}_{A}$. Assume, by contradiction, that $\left\{f_{i}^{\prime}\right\}_{1}^{n} \notin \mathcal{F}_{A}$. Thus there exists a set $A^{\prime}$ of the form $A \cap B$ for some $B \in \mathcal{B} \backslash \mathcal{M}$ such that $A^{\prime}=\bigcup A_{i}^{\prime}$, $A_{i}^{\prime}$ are pairwise disjoint and $f_{i}^{\prime} \mid A_{i}^{\prime} \in \mathrm{C}^{<\mathfrak{c}}\left(A_{i}^{\prime}\right)$. Let us denote $\bigcup\left(f_{i}^{\prime} \mid A_{i}^{\prime}\right)$ by $f^{\prime}$. Note 
that $A^{\prime} \subseteq^{*} A_{\max }$. Since $g_{1} \in \mathrm{SZ}\left(A_{1}\right)$, we have $\left|A_{1} \cap A_{1}^{\prime}\right|<\mathfrak{c}$. This observation and Martin's Axiom imply that $A_{1} \cap A_{1}^{\prime} \in \mathcal{M}$. So we may assume $A_{1} \cap A_{1}^{\prime}=\emptyset$. Then $f^{\prime} \mid\left(A_{1} \cap A^{\prime}\right) \subseteq \bigcup_{i=2}^{n} f_{i}$. This implies that $f^{\prime}\left|\left(A_{1} \cap A^{\prime}\right) \cup f\right|\left(\bigcup_{i=2}^{n} A_{i} \cap A^{\prime}\right) \in \mathrm{C}^{<\mathfrak{c}}\left(A^{\prime}\right)$. Hence $\bigcup_{i=2}^{n} f_{i}$ contains a function from $\mathrm{C}^{<\mathfrak{c}}\left(A^{\prime}\right)$. So $\left\{f_{i}\right\}_{2}^{n} \notin \mathcal{F}_{A}$, a contradiction.

Let us make here a comment about Lemma 2.2.2. One could expect the lemma to hold for bigger families of functions. However, Lemma 2.2.2 cannot be generalized for infinite families of functions. It is implied by the following counterexample.

Example 2.2.3 (CH) There exists an infinite family $\left\{f_{n}\right\}_{n<\omega} \subseteq \mathbb{R}^{\mathbb{R}}$ for which the conclusion of Lemma 2.2.2 fails.

Proof. Continuum Hypothesis implies the existence of an Ulam matrix on $\mathbb{R}$, e.g. the family $\left\{M_{\xi}^{n}: n<\omega, \xi<\mathfrak{c}\right\}$ of subsets of $\mathbb{R}$ with

$$
M_{\xi}^{n} \cap M_{\alpha}^{n}=\emptyset, \text { for } n<\omega, \xi<\alpha<\mathfrak{c},
$$

the complement of $\bigcup_{n<\omega} M_{\xi}^{n}$ is a countable set for $\xi<\mathfrak{c}$.

Fix an enumeration $\left\{x_{\xi}: \xi<\mathfrak{c}\right\}$ of $\mathbb{R}$. Define $f_{n}$ as an extension of $\bigcup_{\xi<\mathfrak{c}} x_{\xi} \chi_{M_{\xi}^{n}}$ onto $\mathbb{R}$, for every $n<\omega$. We are now in a position to show that $F=\left\{f_{n}: n<\omega\right\}$ is the counterexample for the conclusion of Lemma 2.2.2. Since every vertical section of $\bigcup F$ is countable and every horizontal section is comeager, it follows that $\bigcup F$ is non-Borel set of second category. Now, let $A_{n} \subseteq \mathbb{R}$ be such that $f_{n} \mid A_{n} \in \operatorname{CC}\left(A_{n}\right)$, for every $n$. Since the graph of a continuous function is meager in $\mathbb{R}^{2}$, we obtain that $\bigcup_{n<\omega} f_{n} \mid A_{n}$ is also meager as a union of countably many meager sets. We conclude from this that there exists a meager horizontal section of $\bigcup_{n<\omega} f_{n} \mid A_{n}$. Therefore the set $\bigcup F \backslash \bigcup_{n<\omega} f_{n} \mid A_{n}$ contains a constant function defined on comeager Borel set. 
Using very similar technique as the above we can prove

Fact 2.2.4 $(\mathrm{CH})$ Either $\operatorname{Add}(\mathrm{AC}, \mathrm{SZ})=\omega$ or $\operatorname{Add}(\mathrm{AC}, \mathrm{SZ})>\mathfrak{c}$.

Proof. Let us assume that $F=\left\{\phi_{\xi}: \xi<\mathfrak{c}\right\} \subseteq \mathbb{R}^{\mathbb{R}}$ witnesses $\operatorname{Add}(\mathrm{AC}, \mathrm{SZ}) \leq \mathfrak{c}$. For every $n<\omega$, define a function $f_{n}^{*}$ as an extention of $\bigcup_{\xi<\mathfrak{c}} \phi_{\xi} \chi_{M_{\xi}^{n}}$ onto $\mathbb{R}$, where $\left\{M_{\xi}^{n}: n<\omega, \xi<\mathfrak{c}\right\}$ is an Ulam matrix. We claim that $\left\{f_{n}^{*}: n<\omega\right\}$ witnesses $\operatorname{Add}(\mathrm{AC}, \mathrm{SZ}) \leq \omega$. To see this fix an $h \in \mathrm{AC}$. By our assumption about $F$, there exists an $\xi_{0}<\mathfrak{c}$ such that $h+f_{\xi_{0}} \notin \mathrm{SZ}$. That means $h+f_{\xi_{0}}$ is continuous on a set $X$ of cardinality continuum. Since $\mathbb{R} \backslash \bigcup_{n<\omega} M_{\xi_{0}}^{n}$ is countable we obtain that $\left|X \cap M_{\xi_{0}}^{m}\right|=\mathfrak{c}$ for some $m<\omega$. Hence $h+f_{m}^{*}$ is continuous on a set of cardinality continuum which means that $h+f_{m}^{*} \notin \mathrm{SZ}$.

\subsection{Proof of the main result}

In this section we prove the main result of this chapter, that is Theorem 2.1.1.

Proof of Theorem 2.1.1 (1): Add(AC, SZ) $\geq \omega$ (under MA).

We begin by fixing $F=\left\{f_{1}, \ldots, f_{n}\right\} \subseteq \mathbb{R}^{\mathbb{R}}$. Let $F^{\prime}=\left\{f_{1}^{\prime}, \ldots, f_{n}^{\prime}\right\} \in \mathcal{F}_{\mathbb{R}}$ be a corresponding family given by Lemma 2.2.2 for $A=\mathbb{R}$. Based on Lemma 2.2.1, we can find a $g \in \mathrm{AC} \cap \mathrm{SZ}$ such that $g+F^{\prime} \subseteq \mathrm{SZ}$. Since $f_{i} \mid\left[f_{i}^{\prime} \neq f_{i}\right] \in \mathrm{C}_{\mathrm{part}}^{<\mathfrak{c}}$ and $g \in \mathrm{SZ}$, we obtain that $g+f_{i} \in \mathrm{SZ}$ (for $i=1,2, \ldots, \mathrm{n}$.)

In order to prove part (2) of Theorem 2.1.1 we need to state one straightforward lemma.

Lemma 2.3.1 $\operatorname{Add}(S Z, D) \leq 2^{<\mathfrak{c}}$. 
Proof. Let us consider the following family $\mathcal{F}^{<\mathfrak{c}}=\left\{r \chi_{A}: A \in[\mathbb{R}]^{<\mathfrak{c}}, r \in \mathbb{Q}\right\}$. Obviously $\left|\mathcal{F}^{<\mathfrak{c}}\right|=2^{<\mathfrak{c}}$. We claim that

$$
\forall_{g \in S Z} g+\mathcal{F}^{<\mathfrak{c}} \not \subset \mathrm{D} \text {. }
$$

To see this, fix $g \in \mathrm{SZ}$. Let $r_{0} \in \mathbb{Q}$ such that $\inf g<r_{0}<\sup g$. Then $g-r_{0} \chi_{A} \notin \mathrm{D}$, where $A=g^{-1}\left[r_{0}\right]$.

Proof of Theorem 2.1.1 (2): $\operatorname{Add}(\mathrm{SZ}, \mathrm{AC})=\operatorname{Add}(\mathrm{SZ}, \mathrm{D})=\mathfrak{c}($ under MA).

Since $\operatorname{Add}(S Z, A C) \leq \operatorname{Add}(S Z, D)$ and $\operatorname{Add}(S Z, D) \leq 2^{<\mathfrak{r}}=\mathfrak{c}$ (assuming MA), it is sufficient to prove that for every family $F \subseteq \mathbb{R}^{\mathbb{R}}$ of cardinality less than $\mathfrak{c}$ there exists a Sierpiński-Zygmund function $h: \mathbb{R} \rightarrow \mathbb{R}$ satisfying the property $h+F \subseteq$ AC.

Let $F=\left\{f_{\xi}: \xi<\kappa\right\} \subseteq \mathbb{R}^{\mathbb{R}}(\kappa=|F|<\mathfrak{c})$ and $\left\{A_{\xi}: \xi<\kappa\right\}$ be a partition of $\mathbb{R}$ into Bernstein sets. By Lemma 2.2.2, for every $\xi<\kappa$ we can find a function $f_{\xi}^{\prime}$ such that the singleton $\left\{f_{\xi}^{\prime}\right\}$ belongs to $\mathcal{F}_{A_{\xi}}$ and $f_{\xi}^{\prime} \mid\left[f_{\xi}^{\prime} \neq f_{\xi}\right] \in \mathrm{C}_{\text {part }}^{<\mathfrak{c} \text {. Now, applying }}$ Lemma 2.2.1 for every $\xi<\kappa$ we obtain a sequence $\left\langle g_{\xi}: A_{\xi} \rightarrow \mathbb{R} \mid \xi<\kappa\right\rangle$ for which the following holds

$$
g_{\xi}+f_{\xi}^{\prime} \in \mathrm{SZ}_{\mathrm{part}} \text { and any extension of } g_{\xi} \text { on } \mathbb{R} \text { is in } \mathrm{AC} \text {, for } \xi<\kappa \text {. }
$$

Since $f_{\xi}^{\prime}\left[\left[f_{\xi}^{\prime} \neq f_{\xi}\right] \in \mathrm{C}_{\text {part }}^{<\mathfrak{c}}\right.$ and $\mathrm{SZ}(X)+\mathrm{C}^{<\mathfrak{c}}(X)=\mathrm{SZ}(X)$ for every $X \subseteq \mathbb{R}$, we conclude that $g_{\xi}+f_{\xi} \in \mathrm{SZ}_{\mathrm{part}}, \xi<\kappa$. Put $h=\bigcup_{\xi<\kappa}-\left(g_{\xi}+f_{\xi}\right)$. Since Martin's Axiom implies the regularity of $\mathfrak{c}$ we obtain that $h \in \mathrm{SZ}$. Clearly, $h+F \subseteq \mathrm{AC}$.

As a remark let us notice that parts (1) and (2) of the main result, as well as Lemma 2.2.1 and Lemma 2.2.2, could be proved under weaker assumptions. The proofs require only two consequences of Martin's Axiom: $\mathfrak{c}=\mathfrak{c}^{<\mathfrak{c}}$ (this implies regularity of $\mathfrak{c}$ ); the union of less than $\mathfrak{c}$-many meager sets is meager. 
Proof of Theorem 2.1.1 (3): If the theory "ZFC $+\exists$ measurable cardinal" is consistent then so is "ZFC $+\operatorname{Add}(\mathrm{AC}, \mathrm{SZ})>\mathfrak{c}>\omega_{1} . "$

We will show that the existence of $\mathfrak{c}$-additive $\sigma$-saturated ideal $\mathcal{J}$ in $P(\mathbb{R})$ containing $\mathcal{M}$ implies $\operatorname{Add}(\mathrm{AC}, \mathrm{SZ})>\mathfrak{c}$. It is known that the existence of such an ideal is equiconsistent with "ZFC $+\exists$ measurable cardinal." ${ }^{1}$ (See [13].)

First notice that we may assume that $\mathcal{J} \cap \mathcal{B}=\mathcal{M}$. To see this suppose that there exists a Borel set $B$ of second category in $\mathcal{J}$. $B$ is residual in some open interval $I$. Then $I \in \mathcal{J}$ because $I \backslash B$ is meager and $I=(B \cap I) \cup(I \backslash B)$. Now, let $U$ be a maximal open set belonging to $\mathcal{J}$. Such a set exists because the union of all open sets from $\mathcal{J}$ can be represented as a union of countable many such sets. We have that $\mathbb{R} \backslash U$ contains a nonempty open interval $I_{0}$. Otherwise it would be nowhere-dense and then $\mathbb{R}=U \cup(\mathbb{R} \backslash U) \in \mathcal{J}$. Now, any homeomorphism between $I_{0}$ and $\mathbb{R}$ induces the desired ideal on $\mathbb{R}$.

The schema of the proof is similar to the idea of combining Lemmas 2.2.1 and 2.2.2 in the proof of $\operatorname{Add}(\mathrm{AC}, \mathrm{SZ}) \geq \omega$. First step is to show that

$(*)$ for each $f: \mathbb{R} \rightarrow \mathbb{R}$ there exists an $f^{\mathcal{J}} \in \mathbb{R}^{\mathbb{R}}$ such that $f \mid\left[f \neq f^{\mathcal{J}}\right] \in \mathrm{CC}_{\text {part }}$ and $f^{\mathcal{J}} \mid X \notin \mathrm{CC}(X)$ for every $X \notin \mathcal{J}$.

To see this fix an $f \in \mathbb{R}^{\mathbb{R}}$. We claim that there exists a set $Y$ such that $f \mid Y \in$ $\mathrm{CC}(Y)$ and $Y^{\prime} \subseteq^{\mathcal{J}} Y$ for all $Y^{\prime}$ satisfying $f \mid Y^{\prime} \in \mathrm{CC}\left(Y^{\prime}\right)$, where $\subseteq^{\mathcal{J}}$ is defined by

$$
Z_{1} \subseteq^{\mathcal{J}} Z_{2} \text {, if } Z_{1} \backslash Z_{2} \in \mathcal{J} \text {. }
$$

\footnotetext{
${ }^{1}$ The desired model is obtained by adding $\kappa$-many Cohen reals, where $\kappa$ is a measurable cardinal in the ground model.
} 
If the claim did not hold then we could easily construct a strictly increasing (in terms of $\subseteq^{\mathcal{J}}$ ) uncountable sequence of subsets of $\mathbb{R}$. Indeed, assume that the desired sequence of sets $X_{\xi}$ is defined for all $\xi<\alpha$, where $\alpha<\omega_{1}$. Note that $f \mid \bigcup_{\xi<\alpha} X_{\xi} \in$ $\mathrm{CC}_{\text {part }}$. By assumption there exists a set $X$ such that $\bigcup_{\xi<\alpha} X_{\xi} \subseteq \mathcal{J} X \nsubseteq^{\mathcal{J}} \bigcup_{\xi<\alpha} X_{\xi}$ and $f \mid X \in \mathrm{CC}_{\text {part }}$. We set $X_{\alpha}=X$. Thus by transfinite induction the sequence is defined for all $\alpha<\omega_{1}$. But the existence of this sequence would imply the existence of an uncountable family of disjoint sets outside of $\mathcal{J}$ which contradicts the fact that $\mathcal{J}$ is $\sigma$-saturated.

So we proved that the set $Y$ exists. Now put $f^{\mathcal{J}}=f \mid(\mathbb{R} \backslash Y) \cup g$, where $g$ is any function from $\mathrm{SZ}(Y)$. Clearly, $f^{\mathcal{J}}$ is the desired function from $(*)$.

In the next step we fix a family $F$ of real functions of cardinality $\mathfrak{c}$. Let $F=$ $\left\{h_{\xi}: \xi<\mathfrak{c}\right\}$ be an enumeration of $F$ and $\left\langle f_{\alpha}: \alpha<\mathfrak{c}\right\rangle$ be a sequence of all continuous functions defined on $G_{\delta}$ subsets of $\mathbb{R}$. Based on the previous reasoning we may assume that $h_{\xi} \mid X \notin \mathrm{CC}(X)$ for every $X \notin \mathcal{J}$ and $\xi<\mathfrak{c}$. Notice that if $\gamma, \alpha<\mathfrak{c}$ and $f_{\alpha} \mid X \subseteq$ $\bigcup_{\xi, \beta<\gamma}\left(f_{\xi}-h_{\beta}\right)$ then $X \in \mathcal{J}$. This is so since $X \subseteq \bigcup_{\xi, \beta<\gamma}\left[f_{\alpha}=f_{\xi}-h_{\beta}\right]$ and every set $\left[f_{\alpha}=f_{\xi}-h_{\beta}\right]=\left[h_{\beta}=f_{\xi}-f_{\alpha}\right] \in \mathcal{J}$. Consequently, the set $\operatorname{dom}\left(f_{\alpha} \backslash \bigcup_{\xi, \gamma<\alpha}\left(f_{\xi}-h_{\gamma}\right)\right)$ does not belong to $\mathcal{J}$ provided $\operatorname{dom}\left(f_{\alpha}\right) \notin \mathcal{J}$.

Now we construct a sequence $\left\langle g_{\xi}: \xi<\mathfrak{c}\right\rangle$ of partial functions such that $g_{\alpha}$ is a countable dense subset of $f_{\alpha} \backslash \bigcup_{\xi, \gamma<\alpha}\left(\left(f_{\xi}-h_{\gamma}\right) \cup f_{\xi} \cup L\left(D_{\xi}\right)\right)$ for $\alpha<\mathfrak{c}$, where $D_{\gamma}=\operatorname{dom}\left(g_{\gamma}\right)$.

The same kind of argument as in the proof of Lemma 2.2.1 (i)\&(ii) shows that $g^{\prime}=\bigcup_{\xi<\mathfrak{c}} g_{\xi}$ is in $\mathrm{SZ}_{\mathrm{part}}$ and intersects every blocking set. So if $g$ is any SierpińskiZygmund extension of $g^{\prime}$ then $g \in \mathrm{AC}$ and $g+F \subseteq \mathrm{SZ}$. 
Proof of Theorem 2.1.1 (4): Add $(\mathrm{PC}, \mathrm{SZ})=\mathrm{A}(\mathrm{SZ})$ and $\mathrm{Add}(\mathrm{SZ}, \mathrm{PC})=2^{\mathfrak{c}}$.

First we prove the equality $\mathrm{Add}(\mathrm{PC}, \mathrm{SZ})=\mathrm{A}(\mathrm{SZ})$. In order to do it we need the following easy lemma.

Lemma 2.3.2 For every function $f \in \mathbb{R}^{\mathbb{R}}$ there is a function $f^{\prime} \in \mathrm{PC}$ such that $\left|\left[f \neq f^{\prime}\right]\right| \leq \omega$.

ProOf. Let $g: \mathbb{Q} \rightarrow \mathbb{Q}$ be a function with dense graph. Then $f^{\prime}=g \cup f \mid(\mathbb{R} \backslash \mathbb{Q})$ is the required function.

Now, to show $\operatorname{Add}(\mathrm{PC}, \mathrm{SZ})=\mathrm{A}(\mathrm{SZ})$, note that $\operatorname{Add}(\mathrm{PC}, \mathrm{SZ}) \leq \operatorname{Add}\left(\mathbb{R}^{\mathbb{R}}, \mathrm{SZ}\right)=$ $\mathrm{A}(\mathrm{SZ})$. What is left to prove is that $\mathrm{Add}(\mathrm{PC}, \mathrm{SZ}) \geq \mathrm{A}(\mathrm{SZ})$. Let $F \subseteq \mathbb{R}^{\mathbb{R}}$ be a family of cardinality less than A(SZ). So there exists a function $g \in \mathbb{R}^{\mathbb{R}}$ such that $g+F \subseteq$ SZ. Let $g^{\prime} \in \mathrm{PC}$ be a function obtained from $g$ by applying Lemma 2.3.2. Since every Sierpiński-Zygmund function modified on a set of cardinality less than $\mathfrak{c}$ remains Sierpiński-Zygmund, it is easy to see that $g^{\prime}+F \subseteq \mathrm{SZ}$.

Before we start proving that $\operatorname{Add}(\mathrm{SZ}, \mathrm{PC})=2^{\mathfrak{c}}$, we introduce the following

Definition 2.3.3 A set $X \subseteq \mathbb{R}^{2}$ is called Sierpiński-Zygmund set (shortly SZ-set), if for every partial real continuous function $f$ we have $|f \cap X|<\mathfrak{c}$.

An argument, similar to the one used in proving the existence of Sierpiński-Zygmund function, leads to

Lemma 2.3.4 There exists an SZ-set $X \subseteq \mathbb{R}^{2}$ such that $\left|\mathbb{R} \backslash X_{x}\right|<\mathfrak{c}$ for every $x \in \mathbb{R}$, where $X_{x}=\{y \in \mathbb{R}:\langle x, y\rangle \in X\}$. 
Proof. Let $\left\langle x_{\alpha}: \alpha<\mathfrak{c}\right\rangle$ and $\left\langle f_{\alpha}: \alpha<\mathfrak{c}\right\rangle$ be the sequences of all real numbers and all continuous functions defined on a $G_{\delta}$ subset of $\mathbb{R}$, respectively. We will define the set $X$ by defining its vertical sections by transfinite induction. For every $\alpha<\mathfrak{c}$ we put

$$
X_{x_{\alpha}}=\mathbb{R} \backslash\left\{f_{\xi}\left(x_{\alpha}\right): \xi<\alpha\right\} .
$$

Define $X=\bigcup_{\alpha<\mathfrak{c}}\left\{x_{\alpha}\right\} \times X_{x_{\alpha}}$. It is obvious that $X$ has the required properties.

Corollary 2.3.5 There exists a family $\left\{Q_{x} \subseteq \mathbb{R}: x \in \mathbb{R}\right\}$ of pairwise disjoint countable dense sets such that $\bigcup \prod_{x \in \mathbb{R}} Q_{x}$ is an SZ-set.

The next lemma is proved in [7].

Lemma 2.3.6 [7, Lemma 2.2] If $B \subseteq \mathbb{R}$ has cardinality $\mathfrak{c}$ and $H \subseteq \mathbb{Q}^{B}$ is such that $|H|<2^{\mathfrak{c}}$ then there is a $g \in \mathbb{Q}^{B}$ such that $h \cap g \neq \emptyset$ for every $h \in H$.

We give more general version of this lemma.

Lemma 2.3.7 If $B \subseteq \mathbb{R}$ has cardinality $\mathfrak{c}$ and $H \subseteq \prod_{x \in B} Q_{x}$ is such that $|H|<2^{\mathfrak{c}}$ then there is a $g \in \prod_{x \in B} Q_{x}$ such that $h \cap g \neq \emptyset$ for every $h \in H$.

Proof. For every $x \in B$ let $f_{x}: Q_{x} \rightarrow \mathbb{Q}$ be a bijection. Now, for each $h \in H$ we define $h^{\prime}$ as follows

$$
h^{\prime}(x)=f_{x}(h(x)) \text { for all } x \in B .
$$

The family $H^{\prime}=\left\{h^{\prime}: h \in H\right\} \subseteq \mathbb{Q}^{B}$ has cardinality less than $2^{\mathfrak{c}}$. Thus, by Lemma 2.3.6, there is a function $g^{\prime} \in \mathbb{Q}^{B}$ intersecting every element of $H^{\prime}$. Put $g(x)=f_{x}^{-1}\left(g^{\prime}(x)\right)$, for all $x \in B$. It is clear that $g \in \prod_{x \in B} Q_{x}$ and $h \cap g \neq \emptyset$ for every $h \in H$. 
Next we show $\operatorname{Add}(\mathrm{SZ}, \mathrm{PC})=2^{\mathfrak{c}}$. The proof follows the idea of the proof of $[7$, Theorem 1.7 (3)]. Let $F \subseteq \mathbb{R}^{\mathbb{R}}$ be such that $|F|<2^{\mathfrak{c}}$. We will find a $g \in$ SZ satisfying the condition $g+F \subseteq \mathrm{PC}$.

Let $\mathcal{G}$ be the family of all triples $\langle I, p, m\rangle$ where $I$ is a nonempty open interval with rational end-points, $p \in \mathbb{Q}$, and $m<\omega$. For each $\langle I, p, m\rangle \in \mathcal{G}$ define a set $B_{\langle I, p, m\rangle} \subseteq I$ of size $\mathfrak{c}$ such that $B_{\langle I, p, m\rangle} \cap B_{\langle J, q, n\rangle}=\emptyset$ for any distinct $\langle I, p, m\rangle$ and $\langle J, q, n\rangle$ from $\mathcal{G}$.

Let $\langle I, p, m\rangle \in \mathcal{G}$ be fixed. For each $f \in F$ choose $h_{\langle I, p, m\rangle}^{f} \in \prod_{x \in B_{\langle I, p, m\rangle}} Q_{x}$ such that

$$
\left|p-\left(f(x)+h_{\langle I, p, m\rangle}^{f}(x)\right)\right|<\frac{1}{m} \text { for every } x \in B_{\langle I, p, m\rangle} .
$$

Then, by Lemma 2.3.7 used with a set $H_{\langle I, p, m\rangle}=\left\{h_{\langle I, p, m\rangle}^{f}: f \in F\right\}$ used in place $H$, there exists a $g_{\langle I, p, m\rangle} \in \prod_{x \in B_{\langle I, p, m\rangle}} Q_{x}$ such that

$$
\forall f \in F \exists x \in B_{\langle I, p, m\rangle} h_{\langle I, p, m\rangle}^{f}(x)=g_{\langle I, p, m\rangle}(x) .
$$

Now, let $g \in \prod_{x \in \mathbb{R}} Q_{x}$ be a common extension of all functions $g_{\langle I, p, m\rangle}$. Since, by Corollary 2.3.5, $\cup \prod_{x \in \mathbb{R}} Q_{x}$ is an SZ-set we conclude that $g$ is of Sierpiński-Zygmund type. The function $g$ has also the following property. For every $\langle I, p, m\rangle \in \mathcal{G}$ and every $f \in F$ there exists $x \in B_{\langle I, p, m\rangle} \subseteq I$ such that

$$
|p-(f(x)+g(x))|<\frac{1}{m} .
$$

So for each $f \in F$, the function $f+g$ is dense in $\mathbb{R}^{2}$. Thus $f+g \in \mathrm{PC}$. 


\subsection{Sierpiński-Zygmund sets on the plane}

Let us recall that a set $X \subseteq \mathbb{R}^{2}$ is Sierpiński-Zygmund (SZ-set) if it does not contain any partial continuous function with the domain of cardinality continuum $\mathfrak{c}$. (See Definition 2.3.3.) We denote the family of all SZ-sets by $\mathcal{J}_{S Z}$. Since every SierpińskiZygmund function is also an SZ-set we have that $\mathcal{J}_{S Z}$ is not empty.

The next fact follows directly from the definition.

Fact 2.4.1 $\mathcal{J}_{S Z}$ is a $\mathrm{cf}(\mathfrak{c})$-additive ideal.

Proof. Take a $\kappa<\operatorname{cf}(\mathfrak{c})$. Let $\left\{X_{\xi}: \xi<\kappa\right\} \subseteq \mathcal{J}_{S Z}$ and $f \subseteq \bigcup_{\xi<\kappa} X_{\xi}$ be a partial continuous function. Since $X_{\xi}$ is SZ-set, we have that $\left|f \cap X_{\xi}\right|<\mathfrak{c}$ for each $\xi<\kappa$. Consequently, $\left|f \cap \bigcup_{\xi<\kappa} X_{\xi}\right|=\left|\bigcup_{\xi<\kappa}\left(f \cap X_{\xi}\right)\right|<\mathfrak{c}$.

The question that one could ask here is how "big" an SZ-set can be. An example of the SZ-set that can be considered "big" in some sense is given by Lemma 2.3.4. Observe that the complement of every vertical section of the set $X$ from Lemma 2.3.4 has size less than $\mathfrak{c}$. In particular, if MA holds then every vertical section is residual in $\mathbb{R}$. Moreover, under $\mathrm{CH}$, the complement of every vertical section of $X$ is countable. It turns out that the existence of such SZ-set (i.e., with co-countable vertical sections) is equivalent to $\mathrm{CH}$. We state

Proposition 2.4.2 $\mathrm{CH}$ is equivalent to the existence of a $S Z$-set $X \subseteq \mathbb{R}^{2}$ with the following property

$$
\left|\mathbb{R} \backslash X_{x}\right| \leq \omega \text { for every } x \in \mathbb{R} \text {. }
$$

Proof. The existence of the desired set under the assumption of $\mathrm{CH}$ follows from the previous discussion. So we need to prove the opposite implication. Assume, by 
the way of contradiction, that the desired set $X$ exists and $\mathrm{CH}$ does not hold, e.g. $\mathfrak{c}>\omega_{1}$. Since $X$ is an SZ-set we get

(*) $X^{y}=\{x \in \mathbb{R}:\langle x, y\rangle \in X\}$ has cardinality less than $\mathfrak{c}$ for every $y \in \mathbb{R}$.

We claim that there exists an $A \in[\mathbb{R}]^{\omega_{1}}$ such that $\left|\bigcup_{y \in A} X^{y}\right|<\mathfrak{c}$. The following two cases are possible.

Case 1. There exists a $\kappa<\mathfrak{c}$ such that $Z_{\kappa}=\left\{y:\left|X^{y}\right|=\kappa\right\}$ is uncountable.

Then we choose $A \in\left[Z_{\kappa}\right]^{\omega_{1}}$. Obviously, $\left|\bigcup_{y \in A} X^{y}\right|=\kappa \omega_{1}<\mathfrak{c}$.

Case 2. $\left|Z_{\kappa}\right| \leq \omega$ for every cardinal $\kappa<\mathfrak{c}$.

Put $Z=\left\{\left|X^{y}\right|: y \in \mathbb{R}\right\}$ and observe that $\mathbb{R}=\bigcup_{\kappa \in Z} Z_{\kappa} .(*)$ implies that if $\kappa \in Z$ then $\kappa<\mathfrak{c}$. Consequently, since the union of less than continuum many countable sets has size less than continuum, we conclude that $|Z|=\mathfrak{c}$. Let $\lambda$ be the $\omega_{1}$-st element of $Z$. We define $A=\left\{y:\left|X^{y}\right|<\lambda\right\}$. Clearly, $\left|\bigcup_{y \in A} X^{y}\right|=\left|\bigcup_{\kappa<\lambda} Z_{\kappa}\right| \leq \lambda \omega<\mathfrak{c}$.

Now choose an $x \in \mathbb{R} \backslash \bigcup_{y \in A} X^{y}$ and notice that $(\{x\} \times A) \cap X=\emptyset$. So $A \subseteq \mathbb{R} \backslash X_{x}$. This is in contradiction with the fact that every vertical section of $X$ is co-countable.

It is worth remarking here that SZ-sets with the Baire property or measurable are "small." It means that every measurable SZ-set has measure zero and every SZ-set with the Baire property is meager. This follows from Fubini Theorem and Kuratowski-Ulam Theorem, respectively. But do such "small" SZ-sets exist? The answer is positive. It is easy to construct a Sierpiński-Zygmund function (so also an SZ-set) contained in $\mathbb{R} \times \mathfrak{C}$, whose domain is the whole real line. $\mathfrak{C}$ is the standard linear Cantor set. Observe also that there are "big" SZ-sets in terms of outer measure. The set $X$ from Lemma 2.3.4 is of full outer measure. To see this, choose a closed set 
$F \subseteq \mathbb{R}^{2} \backslash X$. Based on the properties of $X$ we conclude that every vertical section of $F$ is countable. Hence $F$ is of measure zero. This proves that $X$ is of full outer measure.

The above discussion states that "good" SZ-sets (in terms of measure or Baire property) are "small". However, we have the following

Remark 2.4.3 There exists an SZ-set which is Marczewski measurable but not Marczewski null.

ProOf. Recall that a set $M \subseteq \mathbb{R}^{n}$ is Marczewski measurable is for every perfect set $P$ there is a perfect subset $Q$ of $P$ such that $Q \subseteq M$ or $Q \cap M=\emptyset$. $M$ is Marczewski null if the second condition holds for every $P$.

We claim that the set $X$ from Lemma 2.3.4 is the desired set. Let us see why $X$ is Marczewski measurable but not Marczewski null. Fix a perfect set $P \subseteq \mathbb{R}^{2}$. There are two possible cases. Either some vertical section $P_{a}$ of $P$ is perfect, or all vertical sections are countable. In the first case, there is a $Q \subseteq\{a\} \times P_{a}$ completely contained in $X$, because the complement of every vertical section of $X$ has cardinality less than $\mathfrak{c}$. In the second case, we can find a partial continuous function $f \subseteq P$ defined on a perfect set. To see this consider a function $g: \operatorname{dom}(P) \rightarrow \mathbb{R}$ defined by $g(x)=\sup \left(P_{x} \cap(-\infty, 0]\right)$. The function $g$ is upper semi-continuous so also of Baire class one. Thus, $g$ contains a continuous function defined on a perfect set. (See [19].)

Since $|f \cap X|<\mathfrak{c}$, the restriction of $f$ to some perfect subset $R$ of $\operatorname{dom}(f)$ is disjoint with $X$. Note that $f \mid R$ is a perfect set. Thus $P$ contains a perfect subset disjoint with $X$. This completes the proof of our remark.

Another interesting observation is that the property of being an SZ-set is not 
preserved under the homeomorphic images. It is easy to see that any vertical line is an SZ-set, but after a rotation, for example about $\frac{\pi}{4}$, it is not an SZ-set any more. However, if $h: \mathbb{R}^{2} \rightarrow \mathbb{R}^{2}$ is a homeomorphism preserving vertical lines then $h[X]$ is an SZ-set for every $X \in \mathcal{J}_{S Z}$.

Fact 2.4.4 Let $h: \mathbb{R}^{2} \rightarrow \mathbb{R}^{2}$ be an homeomorphism such that $h[L]$ is a vertical line for every vertical line $L$. Then $h\left\{\mathcal{J}_{S Z}\right\}=\left\{h[X]: X \in \mathcal{J}_{S Z}\right\}=\mathcal{J}_{S Z}$.

Proof. First we show the inclusion $h\left\{\mathcal{J}_{S Z}\right\} \subseteq \mathcal{J}_{S Z}$. It is easy to see that if $f: A \rightarrow \mathbb{R}$ is a partial continuous function then $h^{-1}[f]: A \rightarrow \mathbb{R}$ is also continuous. This implies that for every $X \in \mathcal{J}_{S Z}, h[X]$ is also in $\mathcal{J}_{S Z}$.

Now to show the other inclusion, let us fix a $Y \in \mathcal{J}_{S Z}$. Note that $h^{-1}$ also preserves all vertical lines. Thus, from the first part of the proof, $X=h^{-1}[Y] \in \mathcal{J}_{S Z}$. Hence $Y=h[X] \in h\left\{\mathcal{J}_{S Z}\right\}$.

Definition 2.4.5 A set $X \subseteq \mathbb{R}^{2}$ is called SZ-shiftable, if there exists a function $f: \mathbb{R} \rightarrow \mathbb{R}$ such that $f+X$ is SZ-set.

We denote the family of all SZ-shiftable sets by $\mathcal{S Z}_{\text {shift }}$. Obviously $\mathcal{J}_{S Z} \subseteq \mathcal{S} \mathcal{Z}_{\text {shift }}$, so $\mathcal{S Z}_{\text {shift }}$ is not empty.

Lemma 2.4.6 Let $X \subseteq \mathbb{R}^{2}$. If for all $x \in \mathbb{R}$ and $A \in[\mathbb{R}]^{<\mathfrak{c}}$ there exists an $a \in \mathbb{R}$ such that $(a+A) \cap X_{x}=\emptyset$, then $A$ is SZ-shiftable.

Proof. Let $\left\langle x_{\alpha}: \alpha<\mathfrak{c}\right\rangle$ and $\left\langle f_{\alpha}: \alpha<\mathfrak{c}\right\rangle$ be the sequences of all real numbers and all continuous functions defined on a $G_{\delta}$ subset of $\mathbb{R}$, respectively. We will define a function $f: \mathbb{R} \rightarrow \mathbb{R}$ which shifts $X$ into $\mathcal{J}_{S Z}$, using transfinite induction. For 
every $\alpha<\mathfrak{c}$ we choose $f\left(x_{\alpha}\right) \in \mathbb{R}$ such that $\left(f\left(x_{\alpha}\right)+X_{x_{\alpha}}\right) \cap\left\{f_{\xi}\left(x_{\alpha}\right): \xi<\alpha\right\}=\emptyset$. Such a choice is possible because of the assumptions on $X$. It is easy to see that $\operatorname{dom}\left((f+X) \cap f_{\beta}\right) \subseteq\left\{x_{\xi}: \xi<\beta\right\}$ for each $\beta<\mathfrak{c}$. Thus $f+X \in \mathcal{J}_{S Z}$.

Recall that under Martin's Axiom (MA) the union of less than $\mathfrak{c}$ meager sets is meager. Suppose that $A \in[\mathbb{R}]^{<\mathfrak{c}}$ and $B \subseteq \mathbb{R}$ is meager. Then the set $B-A=$ $\bigcup_{x \in A}(B-x)$ is meager as a union of less than $\mathfrak{c}$ meager sets. Now, if we choose an $a \notin B-A$ then $(a+A) \cap B=\emptyset$. Notice that the same argument can be repeated for the sets of measure zero.

The above discussion and Lemma 2.4.6 immediately imply

Corollary 2.4.7 (MA) If each vertical section of a set $X \subseteq \mathbb{R}^{2}$ is meager or of measure zero, then $X \in \mathcal{S Z}_{\text {shift }}$.

It may also be of interest to determine whether $\mathcal{S Z}_{\text {shift }}$ is closed under the union operation. Fact 2.4.1 states, in particular, that the union of two SZ-sets is also an SZ-set. Thus, the natural question that appears here is whether the same is true for SZ-shiftable sets. It turns out not to be the case.

Example 2.4.8 There exist $A_{1}, A_{2} \in \mathcal{S} \mathcal{Z}_{\text {shift }}$ such that $A_{1} \cup A_{2}=\mathbb{R}^{2} \notin \mathcal{S} \mathcal{Z}_{\text {shift }}$.

Proof. Put $A_{1}$ to be the set $X$ from Lemma 2.3.4 and $A_{2}$ to be its complement. Based on Lemma 2.4.6 $A_{2}$ is SZ-shiftable. Next, notice that $A_{1} \in \mathcal{J}_{S Z} \subseteq \mathcal{S Z}_{\text {shift }}$. Finally, $A_{1} \cup A_{2}=\mathbb{R}^{2}$ and obviously $\mathbb{R}^{2}$ is not in $\mathcal{S Z}_{\text {shift }}$.

Before we finish this section let us make a comment about Theorem 2.1.1 (1). Note that this result can be expressed using the notion of SZ-sets. Under MA the following holds: 
If, for some fixed $n \in \omega$, every vertical section of the set $X \subseteq \mathbb{R}^{2}$ has at most $n$ elements then there exists an almost continuous function $f: \mathbb{R} \rightarrow \mathbb{R}$ such that $f+X \in \mathcal{J}_{S Z}$.

We generalize this result.

Theorem 2.4.9. (MA) If every vertical section of the set $X \subseteq \mathbb{R}^{2}$ is finite then there exists an almost continuous function $f: \mathbb{R} \rightarrow \mathbb{R}$ such that $f+X \in \mathcal{J}_{S Z}$.

Proof. Let us consider the partition $\left\{H_{n}: n \in \omega\right\}$ of $\mathbb{R}$, where $H_{n}$ is defined by $H_{n}=\left\{x \in \mathbb{R}:\left|X_{x}\right|=n\right\}$. Let $G_{n} \subseteq \mathbb{R}$ be a maximal open set such that $H_{n}$ is everywhere of second category in $G_{n}$. Such a set can be easily constructed. Simply define $G_{n}$ as the interior of the set $\mathbb{R} \backslash \bigcup_{I \in \mathcal{I}_{n}} I$, where $\mathcal{I}_{n}$ is the set of all open intervals in which $H_{n}$ is meager.

We claim that for every $n<\omega$, there exists a function $g_{n}:\left(G_{n} \cap H_{n}\right) \rightarrow \mathbb{R}$ such that $g_{n}+X=\left\{\left\langle x, g_{n}(x)+y\right\rangle: x \in\left(G_{n} \cap H_{n}\right),\langle x, y\rangle \in X\right\} \in \mathcal{J}_{S Z}$ and $\bigcup_{n<\omega} g_{n}$ intersects every blocking set $B$.

First observe that this claim implies the conclusion of the theorem. Put $g: \mathbb{R} \rightarrow \mathbb{R}$ to be an extension of $\bigcup_{n<\omega} g_{n}$ such that $\left[g \mid\left(\mathbb{R} \backslash \bigcup_{n<\omega} G_{n} \cap H_{n}\right)\right]+X$ is an SZ-set. This extension exists based on Corollary 2.4.7. Thus, $g+X$ is the union of countable many SZ-sets. Consequently, $g+X \in \mathcal{J}_{S Z}$. Clearly, $g$ intersects every blocking set, so $g \in \mathrm{AC}$.

To complete the proof we need to show the above claim. Fix an $n<\omega$ and put $A_{n}=\left(G_{n} \cap H_{n}\right) \cup \bigcup_{I \in \mathcal{I}_{n}} I . A_{n}$ is everywhere of second category. Notice also that the part of $X$ contained in $\left(G_{n} \cap H_{n}\right) \times \mathbb{R}$ can be covered by $n$ functions $f_{1}, \ldots, f_{n}$ from $\mathbb{R}$ to $\mathbb{R}$. So, by Lemma 2.2.1 and Lemma 2.2.2, there exists a function $g_{n}^{\prime}: A_{n} \rightarrow \mathbb{R}$ 
such that $g_{n}^{\prime}+\left\{f_{1}, \ldots, f_{n}\right\} \subseteq \mathrm{SZ}_{\text {part }}$ and $\operatorname{dom}\left(g_{n}^{\prime} \cap B\right)$ is dense in some non-empty open interval $I_{B}$ for every blocking set $B$. Thus, if we define $g_{n}=g_{n}^{\prime} \mid\left(G_{n} \cap H_{n}\right)$ then $g_{n}+X \in \mathcal{J}_{S Z}$.

What remains to prove is that $\bigcup_{n<\omega} g_{n}$ intersects every blocking set $B$. Notice that $I_{B} \cap G_{n} \neq \emptyset$ for some $n$. Thus, $g_{n} \cap B \neq \emptyset$. Consequently, $\emptyset \neq B \cap \bigcup_{n<\omega} g_{n} \subseteq$ $B \cap g$. This finishes the proof.

In other words, under MA every planar set $X$ with finite vertical sections can be shifted into $\mathcal{J}_{S Z}$ by an almost continuous function. 


\section{Chapter 3}

\section{Classes related to additive properties}

This chapter is devoted entirely to the concept of additivity and is based on the paper [21]. As said in the preliminaries, the additive functions were defined as the solutions to Cauchy's Functional Equation, which plays a very important role in the theory of functional equations. It is also of interest, similarly as in the case of Darbuox-like functions, to consider functions that "badly" violate the additive condition. That is, functions $f: \mathbb{R} \rightarrow \mathbb{R}$ satisfying the condition $f(x+y) \neq f(x)+f(y)$, for all $x, y \in \mathbb{R}$. We give two examples of families of such functions. In Section 3.1 we define and discuss a class of functions whose graph is a linearly independent set over $\mathbb{Q}$. Then in Section 3.2 we investigate a proper subfamily of this class: functions whose graph is a Hamel basis. Throughout this chapter, $\mathbf{0}$ will denote the zero element of $\mathbb{R}^{n}$. 


\subsection{Functions with linearly independent graphs}

Definition 3.1.1 We say that a function $f: \mathbb{R}^{n} \rightarrow \mathbb{R}$ is linearly independent over $\mathbb{Q}$ (shortly: linearly independent) if $f$ is linearly independent subset of the space $\left\langle\mathbb{R}^{n+1} ; \mathbb{Q} ;+; \cdot\right\rangle$.

The symbol $\operatorname{LIF}\left(\mathbb{R}^{n}\right)$ stands for the family of all linearly independent functions. In the case when $n=1$ we simply write LIF. An easy example shows that the family $\operatorname{LIF}\left(\mathbb{R}^{n}\right)$ is non-empty for all $n \geq 1$.

Example 3.1.2 Every injection from $\mathbb{R}^{n}$ into a Hamel basis $H \subseteq \mathbb{R}$ is linearly independent over $\mathbb{Q}$.

Proof. Let $f: \mathbb{R}^{n} \rightarrow H$ be an injection. Assume that for some $p_{1}, \ldots, p_{n} \in \mathbb{Q}$ and pairwise different $x_{1}, \ldots, x_{n} \in \mathbb{R}^{n}$

$$
\sum_{1}^{n} p_{i}\left\langle x_{i}, f\left(x_{i}\right)\right\rangle=\mathbf{0} .
$$

Since $f\left(x_{1}\right), \ldots, f\left(x_{n}\right) \in H$ are all different and $H$ is linearly independent over $\mathbb{Q}$, we conclude that $p_{1}=\ldots=p_{n}=0$.

As mentioned in the introductory part of this chapter, the linearly independent functions lack the additive property. Thus, $\operatorname{AD}\left(\mathbb{R}^{n}\right) \cap \operatorname{LIF}\left(\mathbb{R}^{n}\right)=\emptyset$.

Below we give some basic properties of the class $\operatorname{LIF}\left(\mathbb{R}^{n}\right)$. Note that Fact 3.1.3 (i) has its counterpart in the case of continuous and Sierpiński-Zygmund functions.

\section{Fact 3.1.3}

(i) $\operatorname{LIF}\left(\mathbb{R}^{n}\right)+\mathrm{AD}\left(\mathbb{R}^{n}\right)=\operatorname{LIF}\left(\mathbb{R}^{n}\right)$. 
(ii) If $f \in \operatorname{LIF}\left(\mathbb{R}^{n}\right)$ then $\left|f\left[\mathbb{R}^{n}\right]\right|=\mathfrak{c}$.

(iii) If $f: \mathbb{R}^{n} \rightarrow \mathbb{R}$ is continuous on some non-empty open set then $f \notin \operatorname{LIF}\left(\mathbb{R}^{n}\right)$.

(iv) $\operatorname{LIF}\left(\mathbb{R}^{n}\right) \cap \mathrm{CC}\left(\mathbb{R}^{n}\right) \neq \emptyset$.

(v) $\mathrm{A}\left(\operatorname{LIF}\left(\mathbb{R}^{n}\right)\right)=\mathfrak{c}$.

Proof. (i) Let $f \in \operatorname{LIF}\left(\mathbb{R}^{n}\right)$ and $g \in \operatorname{AD}\left(\mathbb{R}^{n}\right)$. Fix $x_{1}, \ldots, x_{k} \in \mathbb{R}^{n}$ and $q_{1}, \ldots q_{k} \in$ $\mathbb{Q}$. Now suppose that

$$
\sum_{1}^{k} q_{i}\left\langle x_{i}, f\left(x_{i}\right)+g\left(x_{i}\right)\right\rangle=\mathbf{0} .
$$

Thus, in particular, $\sum_{1}^{k} q_{i} x_{i}=0$. Since $g$ is additive we have $\sum_{1}^{k} q_{i} g\left(x_{i}\right)=0$. Consequently, $\sum_{1}^{k} q_{i}\left\langle x_{i}, f\left(x_{i}\right)\right\rangle=0$. The linear independence of $f$ implies that $q_{1}=$ $\ldots=q_{k}=0$. So $f+g \in \operatorname{LIF}\left(\mathbb{R}^{n}\right)$.

(ii) Notice that it suffices to prove part (ii) for $n=1$. Assume, by the way of contradiction, that $f \in \operatorname{LIF}$ and $|f[\mathbb{R}]|=\kappa<\mathfrak{c}$. We claim that there exist positive $x_{1}, x_{2} \in \mathbb{R}$ with the following properties:

$$
x_{1} \neq x_{2}, f\left(x_{1}\right)=f\left(x_{2}\right) \text {, and } f\left(-x_{1}\right)=f\left(-x_{2}\right) .
$$

To see the claim choose $y_{0} \in \mathbb{R}$ such that $\left|f^{-1}\left(y_{0}\right) \cap(0, \infty)\right|=\kappa^{+}$. Such an element exists because $(0, \infty) \subseteq \bigcup_{y \in \mathbb{R}} f^{-1}(y)$ and $|f[\mathbb{R}]|=\kappa<\mathfrak{c}$. Since $y_{0}$ satisfies the condition $\left|f\left[-f^{-1}\left(y_{0}\right)\right]\right| \leq \kappa<\kappa^{+} \leq\left|-f^{-1}\left(y_{0}\right)\right|$, there exist different $x_{1}, x_{2} \in$ $f^{-1}\left(y_{0}\right) \cap(0, \infty)$ satisfying the equality $f\left(-x_{1}\right)=f\left(-x_{2}\right)$. Note that $x_{1}$ and $x_{2}$ are the required points.

Next observe that

$$
\left\langle x_{1}, f\left(x_{1}\right)\right\rangle+\left\langle-x_{1}, f\left(-x_{1}\right)\right\rangle=\left\langle x_{2}, f\left(x_{2}\right)\right\rangle+\left\langle-x_{2}, f\left(-x_{2}\right)\right\rangle .
$$


This leads to a contradiction with $f \in$ LIF.

(iii) Like in part (ii), it is enough to prove the case $n=1$. Let $(a-h, a+h) \subseteq \mathbb{R}$ be a non-empty open interval such that $f \mid(a-h, a+h)$ is continuous. Consider a function $g:[0, h) \rightarrow \mathbb{R}$ defined by $g(x)=f(a-x)+f(a+x)$. Obviously, $g$ is also continuous. If $g(x)=g(0)=2 f(a)$ for all $x \in[0, h)$ then $f$ is not linearly independent. Hence we may suppose that there exist two different $x_{1}, x_{2} \in(0, h)$ such that $g\left(x_{1}\right)=2 f(a)+p_{1}$ and $g\left(x_{2}\right)=2 f(a)+p_{2}$ for some non-zero rationals $p_{1}, p_{2}$. Then we have

$$
p_{2}\left\langle 2 a, g\left(x_{1}\right)\right\rangle-p_{1}\left\langle 2 a, g\left(x_{2}\right)\right\rangle \in \operatorname{Lin}_{\mathbb{Q}}(\langle 2 a, 2 f(a)\rangle)=\operatorname{Lin}_{\mathbb{Q}}(\langle a, f(a)\rangle) .
$$

Now, recall the definition of $g$ and note $\left\langle a-x_{i}, f\left(a-x_{i}\right)\right\rangle+\left\langle a+x_{i}, f\left(a+x_{i}\right)\right\rangle=$ $\left\langle 2 a, g\left(x_{i}\right)\right\rangle$ for $i=1,2$. Based on (3.1), we see that $f$ is not linearly independent.

(iv) Let us first recall that $\mathbb{R}^{n}$ can be decomposed into $(n+1)$ 0-dimensional spaces $E_{0}, \ldots, E_{n}$. For every perfect set $Q \subseteq \mathbb{R}$ and 0 -dimensional space $E$ there exists an embedding $h_{Q}^{E}: E \rightarrow Q$. (See e.g., [11].) It is also known that there exists a perfect set $P \subseteq \mathbb{R}$ which is linearly independent over $\mathbb{Q}$. (See e.g., [16].) Now, if $P=P_{0} \cup P_{1} \cup \ldots \cup P_{n}$ is a partition of $P$ into $(n+1)$ perfect sets then, by Example 3.1.2, $h_{P_{i}}^{E_{i}}: E_{i} \rightarrow P_{i}$ is a linearly independent subset of $\mathbb{R}^{n+1}$. It is easy to see that $h=\bigcup_{0}^{n} h_{P_{i}}^{E_{i}}: \mathbb{R}^{n} \rightarrow P$ is one-to-one. So, again by Example 3.1.2, $h$ is linearly independent. Obviously, $h \in \mathrm{CC}$.

(v) We start with showing that $\mathrm{A}\left(\operatorname{LIF}\left(\mathbb{R}^{n}\right)\right) \geq \mathfrak{c}$. Let $\mathbb{R}^{n}=\left\{x_{\xi}: \xi<\mathfrak{c}\right\}$. Fix an $F \subseteq \mathbb{R}^{\mathbb{R}^{n}}$ of cardinality less than continuum. We will define, by induction, a function $h: \mathbb{R}^{n} \rightarrow \mathbb{R}$ such that for every $f \in F, h+f$ is one-to-one and $(h+f)\left[\mathbb{R}^{n}\right]$ is linearly independent. Then, by Example 3.1.2, $h+F \subseteq \operatorname{LIF}\left(\mathbb{R}^{n}\right)$. 
Let $\alpha<\mathfrak{c}$. Assume that $h$ is defined on $\left\{x_{\xi}: \xi<\alpha\right\}$, for all $f \in F$ the function $h+f$ is one-to-one, and $(h+f)\left[\left\{x_{\xi}: \xi<\alpha\right\}\right]$ is linearly independent. We will define $h\left(x_{\alpha}\right)$. Choose

$$
h\left(x_{\alpha}\right) \in \mathbb{R} \backslash \operatorname{Lin}_{\mathbb{Q}}\left(\bigcup_{f \in F}\left((h+f)\left[\left\{x_{\xi}: \xi<\alpha\right\}\right] \cup\left\{f\left(x_{\alpha}\right)\right\}\right)\right) .
$$

This choice is possible since

$$
\left|\bigcup_{f \in F}\left((h+f)\left[\left\{x_{\xi}: \xi<\alpha\right\}\right] \cup\left\{f\left(x_{\alpha}\right)\right\}\right)\right|<\mathfrak{c} .
$$

It is easy to see that all the required properties of $h$ are preserved. This ends the proof of $\mathrm{A}\left(\operatorname{LIF}\left(\mathbb{R}^{n}\right)\right) \geq \mathfrak{c}$.

To see the opposite inequality consider $F$ consisting of all constant functions. Then for any function $h: \mathbb{R}^{n} \rightarrow \mathbb{R}$ there is an $f \in F$ such that $h(\mathbf{0})+f(\mathbf{0})=0$. Therefore $h+f \notin \operatorname{LIF}\left(\mathbb{R}^{n}\right)$.

\subsection{Hamel functions}

In this section we confine ourselves to a proper subclass of linearly independent functions. More precisely, we consider the class of Hamel functions. Let us recall that a function $f: \mathbb{R}^{n} \rightarrow \mathbb{R}$ is a Hamel function, $f \in \operatorname{HF}\left(\mathbb{R}^{n}\right)$, if $f$, considered as a subset of $\mathbb{R}^{n+1}$, is a Hamel basis for $\mathbb{R}^{n+1}$. Clearly, $\operatorname{HF}\left(\mathbb{R}^{n}\right) \subseteq \operatorname{LIF}\left(\mathbb{R}^{n}\right)$. A little more challenging argument, comparing with the case of linearly independent functions, proves the existence of a Hamel function. We do not present it here since this observation follows from other results of the current section (e.g., Theorem 3.2.4.)

Fact 3.1.3 states some basic properties of the class $\operatorname{LIF}\left(\mathbb{R}^{n}\right)$. It is interesting whether the same statements are true for $\operatorname{HF}\left(\mathbb{R}^{n}\right)$. Since $\operatorname{HF}\left(\mathbb{R}^{n}\right) \subseteq \operatorname{LIF}\left(\mathbb{R}^{n}\right)$ the 
properties (ii) and (iii) hold trivially. A short additional argument shows that (i) is also true. So we can state

\section{Fact 3.2.1}

(i) $\operatorname{HF}\left(\mathbb{R}^{n}\right)+\mathrm{AD}\left(\mathbb{R}^{n}\right)=\operatorname{HF}\left(\mathbb{R}^{n}\right)$.

(ii) If $f \in \operatorname{HF}\left(\mathbb{R}^{n}\right)$ then $\left|f\left[\mathbb{R}^{n}\right]\right|=\mathfrak{c}$.

(iii) If $f: \mathbb{R}^{n} \rightarrow \mathbb{R}$ is continuous on some nonempty open set then $f \notin \operatorname{HF}\left(\mathbb{R}^{n}\right)$.

Proof. (i) Let $f \in \operatorname{HF}\left(\mathbb{R}^{n}\right)$ and $g \in \operatorname{AD}\left(\mathbb{R}^{n}\right)$. Based on Fact 3.1.3 (i), $f+g$ is linearly independent. Thus, we need to show that $\operatorname{Lin}_{\mathbb{Q}}(f+g)=\mathbb{R}^{n+1}$. Let $v \in \mathbb{R}^{n+1}$ and $v_{x} \in \mathbb{R}^{n}$ be a vector of $n$ first coordinates of $v$. Since $f \in \operatorname{HF}\left(\mathbb{R}^{n}\right)$, there exist $x_{1}, \ldots, x_{n} \in \mathbb{R}^{n}$ and $p_{1}, \ldots, p_{n} \in \mathbb{Q}$ such that

$$
v-\left\langle v_{x},(f+g)\left(v_{x}\right)\right\rangle=\sum_{1}^{n} p_{i}\left\langle x_{i}, f\left(x_{i}\right)\right\rangle .
$$

Note that $\sum_{1}^{n} p_{i} x_{i}=\mathbf{0}$, so $\sum_{1}^{n} p_{i} g\left(x_{i}\right)=g\left(\sum_{1}^{n} p_{i} x_{i}\right)=g(\mathbf{0})=0$. Consequently,

$$
v=\sum_{1}^{n} p_{i}\left\langle x_{i},(f+g)\left(x_{i}\right)\right\rangle+\left\langle v_{x},(f+g)\left(v_{x}\right)\right\rangle .
$$

Hence $v \in \operatorname{Lin}_{\mathbb{Q}}(f+g)$.

However, it remains an open problem whether Fact 3.1.3 (iv) still holds if $\operatorname{LIF}\left(\mathbb{R}^{n}\right)$ is replaced by $\operatorname{HF}\left(\mathbb{R}^{n}\right)$.

Problem 3.2.2 $\mathrm{HF}\left(\mathbb{R}^{n}\right) \cap \mathrm{CC}\left(\mathbb{R}^{n}\right) \neq \emptyset$ ?

But it turns out that the statement of the last part of Fact 3.1.3 is false for the class $\operatorname{HF}\left(\mathbb{R}^{n}\right)$. 
Fact 3.2.3 $\mathrm{A}\left(\operatorname{HF}\left(\mathbb{R}^{n}\right)\right) \leq \omega$ for every $n \in \omega$.

Proof. For each $q \in \mathbb{Q}$ and each open ball $B$ with rational center and radius (rational ball), let us define a function $f_{q}^{B}: \mathbb{R}^{n} \rightarrow \mathbb{R}$ by $f_{q}^{B}=q \chi_{B}$. We claim that for every function $f: \mathbb{R}^{n} \rightarrow \mathbb{R}$ there exist a $q \in \mathbb{Q}$ and a rational ball $B$ such that $f+f_{q}^{B} \notin \operatorname{HF}\left(\mathbb{R}^{n}\right)$. To see this, first note that we may assume that $f=f+f_{0}^{\mathbb{R}^{n}} \in$ $\operatorname{HF}\left(\mathbb{R}^{n}\right)$. Thus, $\langle\mathbf{0}, 1\rangle \in \operatorname{Lin}_{\mathbb{Q}}(f)$. Consequently, there exist $x_{1}, \ldots, x_{k} \in \mathbb{R}^{n}$ and $p_{1}, \ldots, p_{k} \in \mathbb{Q}$ satisfying

$$
\sum_{i=1}^{k} p_{i}\left\langle x_{i}, f\left(x_{i}\right)\right\rangle=\langle\mathbf{0}, 1\rangle
$$

Without loss of generality we may assume that $p_{1} \neq 0$. Now let $q=\frac{-1}{p_{1}}$ and $B$ be a rational ball containing $x_{1}$ but not $x_{2}, \ldots, x_{k}$. It follows easily that $f+f_{q}^{B}$ is not linearly independent over $\mathbb{Q}$. Indeed,

$$
\begin{aligned}
\sum_{i=1}^{k} p_{i}\left\langle x_{i}, f\left(x_{i}\right)+f_{q}^{B}\left(x_{i}\right)\right\rangle & = \\
\sum_{i=1}^{k} p_{i}\left\langle x_{i}, f\left(x_{i}\right)\right\rangle+\sum_{i=1}^{k} p_{i}\left\langle\mathbf{0}, f_{q}^{B}\left(x_{i}\right)\right\rangle & = \\
\langle\mathbf{0}, 1\rangle+p_{1}\langle\mathbf{0}, q\rangle=\langle\mathbf{0}, 1\rangle-\langle\mathbf{0}, 1\rangle & =\mathbf{0} .
\end{aligned}
$$

Notice here that $\mathrm{A}(\mathrm{LIF})=\mathfrak{c}($ Fact $3.1 .3(\mathrm{v})$ ) implies in particular, that every function from $\mathbb{R}^{\mathbb{R}}$ can be written as the algebraic sum of two linearly independent functions. In other words $\operatorname{LIF}+\operatorname{LIF}=\mathbb{R}^{\mathbb{R}}$. Since we just proved that $\mathrm{A}(\mathrm{HF}) \leq \omega$, it would be very interesting to determine whether $\mathrm{HF}+\mathrm{HF}=\mathbb{R}^{\mathbb{R}}$. The following theorem is the main result of this chapter. 
Theorem 3.2.4. Every real function $f \in \mathbb{R}^{\mathbb{R}}$ can be represented as a sum of two Hamel functions. In other words, $\mathbb{R}^{\mathbb{R}}=\mathrm{HF}+\mathrm{HF}$.

Observe that the above theorem and Proposition 1.2.1 (3) \& (5) imply that $\mathrm{A}(\mathrm{HF}) \geq 3$.

Before proving the theorem we introduce some definitions and show auxiliary results. For $f \in \mathbb{R}^{\mathbb{R}}, x \in \mathbb{R}$, and $0<n<\omega$ let

$$
\mathrm{LC}(f, n, x)=\left\{\sum_{1}^{n} p_{i} f\left(x_{i}\right): p_{j} \in \mathbb{Q}, x_{j} \in \mathbb{R}, j=1, \ldots, n, \sum_{1}^{n} p_{i} x_{i}=x\right\} .
$$

When $x=0$ we simply write $\operatorname{LC}(f, n)$. We also use $\operatorname{LC}(f)$ to denote $\bigcup_{0<n<\omega} \operatorname{LC}(f, n)$. Observe that $\operatorname{LC}(f)$ is a linear subspace of $\mathbb{R}$ over $\mathbb{Q}$, that is, $\operatorname{LC}(f)=\operatorname{Lin}_{\mathbb{Q}}(\operatorname{LC}(f))$. This is so because $\operatorname{LC}(f)=\operatorname{Lin}_{\mathbb{Q}}(f) \cap(\{0\} \times \mathbb{R})$.

The sets $\mathrm{LC}(f)$ will play an important role in the proof of Theorem 3.2.4. Thus, we will investigate properties of these sets.

Property 3.2.5 $\mathrm{LC}(f, n) \subseteq \mathrm{LC}(f, 3)+\mathrm{LC}(f, n-1)$ for every $f \in \mathbb{R}^{\mathbb{R}}$ and $3 \leq n<\omega$.

Proof. Let $y \in \operatorname{LC}(f, n)$. So $y=\sum_{1}^{n} p_{i} f\left(x_{i}\right)$ for some $x_{1}, \ldots, x_{n} \in \mathbb{R}$ and $p_{1}, \ldots, p_{n} \in \mathbb{Q}$ satisfying $\sum_{1}^{n} p_{i} x_{i}=0$. Define $x^{\prime}=p_{1} x_{1}+p_{2} x_{2}, q=1$, and $r=-1$. Observe that

$$
p_{1} x_{1}+p_{2} x_{2}+r x^{\prime}=q x^{\prime}+p_{3} x_{3}+\ldots+p_{n} x_{n}=0 .
$$

Thus, $p_{1} f\left(x_{1}\right)+p_{2} f\left(x_{2}\right)+r f\left(x^{\prime}\right) \in \mathrm{LC}(f, 3)$ and $q f\left(x^{\prime}\right)+p_{3} f\left(x_{3}\right)+\ldots+p_{n} f\left(x_{n}\right) \in$ $\mathrm{LC}(f, n-1)$. Since $y=p_{1} f\left(x_{1}\right)+p_{2} f\left(x_{2}\right)+r f\left(x^{\prime}\right)+q f\left(x^{\prime}\right)+p_{3} f\left(x_{3}\right)+\ldots+p_{n} f\left(x_{n}\right)$ we conclude that $y \in \mathrm{LC}(f, 3)+\mathrm{LC}(f, n-1)$. 
Notice that Property 3.2.5 implies that

$$
\text { if }|\mathrm{LC}(f)|=\mathfrak{c} \text { then } m_{0}=\min \{n \in \omega:|\mathrm{LC}(f, n)|=\mathfrak{c}\} \leq 3 \text {. }
$$

Next we show another property which is important for the proof of Theorem 3.2.4. Note that if $\mathfrak{c}$ is regular (i.e., $\operatorname{cf}(\mathfrak{c})=\mathfrak{c}$ ), then the set $Z$ from part (a) can be taken as a singleton.

Property 3.2.6 Assume that $|\mathrm{LC}(f)|=\mathfrak{c}$. Then at least one of the following two cases hold.

(a) There exists a set $Z \in[\mathbb{R}]^{<\mathfrak{c}}$ such that $\left|\bigcup_{z \in Z} \mathrm{LC}(f, 2, z)\right|=\mathfrak{c}$.

(b) For all $X, Y \in[\mathbb{R}]^{<\mathfrak{c}}$ there exist $q_{1}, q_{2}, q_{3} \in \mathbb{Q} \backslash\{0\}$ and pairwise linearly independent $x_{1}, x_{2}, x_{3} \in \mathbb{R}$ such that $\sum_{1}^{3} q_{i} f\left(x_{i}\right) \notin Y, \sum_{1}^{3} q_{i} x_{i}=0$, and $\operatorname{Lin}_{\mathbb{Q}}\left(x_{1}, x_{2}, x_{3}\right) \cap \operatorname{Lin}_{\mathbb{Q}}(X)=\{0\}$.

Proof. Notice first that if $|\mathrm{LC}(f, 2)|=\mathfrak{c}$ then case (a) holds with $Z=\{0\}$. Hence, using (3.2), we may assume that

$$
|\mathrm{LC}(f, 2)|<\mathfrak{c} \text { and }|\mathrm{LC}(f, 3)|=\mathfrak{c} .
$$

Based on the above assumption and the definition of the set $\operatorname{LC}(f, 3)$, we conclude that there exist continuum many triples $\left\langle x_{1}, x_{2}, x_{3}\right\rangle \in \mathbb{R}^{3}$ and $\left\langle p_{1}, p_{2}, p_{3}\right\rangle \in(\mathbb{Q} \backslash\{0\})^{3}$ such that $\sum_{1}^{3} p_{i} x_{i}=0$ and $\sum_{1}^{3} p_{i} f\left(x_{i}\right)$ are all different. Thus, an easy cardinal argument implies the existence of a sequence $\left\langle\left\langle x_{1}^{\xi}, x_{2}^{\xi}, x_{3}^{\xi}\right\rangle \in \mathbb{R}^{3}: \xi<\mathfrak{c}\right\rangle$ and some nonzero rationals $q_{1}, q_{2}, q_{3}$ with the property that $q_{1} x_{1}^{\xi}+q_{2} x_{2}^{\xi}+q_{3} x_{3}^{\xi}=0$ for every $\xi<\mathfrak{c}$, and all $q_{1} f\left(x_{1}^{\xi}\right)+q_{2} f\left(x_{2}^{\xi}\right)+q_{3} f\left(x_{3}^{\xi}\right)$ are different. 
Observe that, if $\operatorname{dim}\left(\left\{x_{1}^{\xi}, x_{2}^{\xi}, x_{3}^{\xi}\right\}\right)=1$ for some $\xi$ then $\operatorname{Lin}_{\mathbb{Q}}\left(x_{1}^{\xi}, x_{2}^{\xi}, x_{3}^{\xi}\right)=\operatorname{Lin}_{\mathbb{Q}}\left(x_{i}^{\xi}\right)$ for some $i \in\{1,2,3\}$. Say $\mathrm{i}=1$. So there is an $s \in \mathbb{Q}$ such that $s q_{1} x_{1}^{\xi}+q_{2} x_{2}^{\xi}=0$. Combining this with the equality $q_{1} x_{1}^{\xi}+q_{2} x_{2}^{\xi}+q_{3} x_{3}^{\xi}=0$ we obtain that $s q_{1} x_{1}^{\xi}+q_{2} x_{2}^{\xi}=$ $(1-s) q_{1} x_{1}^{\xi}+q_{3} x_{3}^{\xi}=0$. Consequently,

$$
\left[s q_{1} f\left(x_{1}^{\xi}\right)+q_{2} f\left(x_{2}^{\xi}\right)\right],\left[(1-s) q_{1} f\left(x_{1}^{\xi}\right)+q_{3} f\left(x_{3}^{\xi}\right)\right] \quad \in \operatorname{LC}(f, 2)
$$

and

$$
\begin{aligned}
q_{1} f\left(x_{1}^{\xi}\right)+q_{2} f\left(x_{2}^{\xi}\right)+q_{3} f\left(x_{3}^{\xi}\right) & = \\
s q_{1} f\left(x_{1}^{\xi}\right)+q_{2} f\left(x_{2}^{\xi}\right)+(1-s) q_{1} f\left(x_{1}^{\xi}\right)+q_{3} f\left(x_{3}^{\xi}\right) & \in \mathrm{LC}(f, 2)+\mathrm{LC}(f, 2) .
\end{aligned}
$$

So if $\operatorname{dim}\left(\left\{x_{1}^{\xi}, x_{2}^{\xi}, x_{3}^{\xi}\right\}\right)=1$ for continuum many $\xi$ then $|\mathrm{LC}(f, 2)|=\mathfrak{c}$. This contradicts (3.3). Thus, we may assume that $\operatorname{dim}\left(\left\{x_{1}^{\xi}, x_{2}^{\xi}, x_{3}^{\xi}\right\}\right)=2$ for all $\xi<\mathfrak{c}$.

Now choose $X, Y \in[\mathbb{R}]^{<\mathfrak{c}}$. Notice that

$(\bullet)$ if $\operatorname{Lin}_{\mathbb{Q}}\left(x_{1}^{\xi}, x_{2}^{\xi}, x_{3}^{\xi}\right) \cap \operatorname{Lin}_{\mathbb{Q}}(X) \neq\{0\}$ and $Z=\operatorname{Lin}_{\mathbb{Q}}(X)$ then

$$
q_{1} f\left(x_{1}^{\xi}\right)+q_{2} f\left(x_{2}^{\xi}\right)+q_{3} f\left(x_{3}^{\xi}\right) \in \bigcup_{z \in Z} \mathrm{LC}(f, 2, z)+\bigcup_{z \in Z} \mathrm{LC}(f, 2, z) .
$$

Indeed, if $\operatorname{Lin}_{\mathbb{Q}}\left(x_{1}^{\xi}, x_{2}^{\xi}, x_{3}^{\xi}\right) \cap \operatorname{Lin}_{\mathbb{Q}}(X) \neq\{0\}$ then there exist $a, b, c \in \mathbb{Q}$ such that $a x_{1}^{\xi}+b x_{2}^{\xi}+c x_{3}^{\xi} \in \operatorname{Lin}_{\mathbb{Q}}(X) \backslash\{0\}$. At least one of the numbers $a, b, c$ is not equal to zero because $a x_{1}^{\xi}+b x_{2}^{\xi}+c x_{3}^{\xi} \neq 0$. Without loss of generality we may suppose that $c \neq 0$ and consequently $c=q_{3}$ (multiply the above equation by $\frac{q_{3}}{c}$.) Then, by subtracting $a x_{1}^{\xi}+b x_{2}^{\xi}+q_{3} x_{3}^{\xi}$ from $q_{1} x_{1}^{\xi}+q_{2} x_{2}^{\xi}+q_{3} x_{3}^{\xi}=0$, we obtain that $\left(q_{1}-a\right) x_{1}^{\xi}+\left(q_{2}-b\right) x_{2}^{\xi} \in \operatorname{Lin}_{\mathbb{Q}}(X) \backslash\{0\}$. So at least one of $\left(q_{1}-a\right),\left(q_{2}-b\right)$ is not 0 . We may assume that $\left(q_{2}-b\right) \neq 0$. (If $\left(q_{1}-b\right) \neq 0$ then the following argument 
works analogously.) Now multiply $\left(q_{1}-a\right) x_{1}^{\xi}+\left(q_{2}-b\right) x_{2}^{\xi}$ by $\frac{q_{2}}{q_{2}-b}$. We get that $r q_{1} x_{1}^{\xi}+q_{2} x_{2}^{\xi} \in \operatorname{Lin}_{\mathbb{Q}}(X)$ and consequently $(1-r) q_{1} x_{1}^{\xi}+q_{3} x_{3}^{\xi}=\left[q_{1} x_{1}^{\xi}+q_{2} x_{2}^{\xi}+q_{3} x_{3}^{\xi}\right]-$ $\left[r q_{1} x_{1}^{\xi}+q_{2} x_{2}^{\xi}\right]=-\left[r q_{1} x_{1}^{\xi}+q_{2} x_{2}^{\xi}\right] \in \operatorname{Lin}_{\mathbb{Q}}(X)$, for some $r \in \mathbb{Q}$. Hence

$$
\left[r q_{1} f\left(x_{1}^{\xi}\right)+q_{2} f\left(x_{2}^{\xi}\right)\right],\left[(1-r) q_{1} f\left(x_{1}^{\xi}\right)+q_{3} f\left(x_{3}^{\xi}\right)\right] \in \bigcup_{z \in Z} \operatorname{LC}(f, 2, z) .
$$

Now the claim $(\bullet)$ follows from

$$
\begin{aligned}
q_{1} f\left(x_{1}^{\xi}\right)+q_{2} f\left(x_{2}^{\xi}\right)+q_{3} f\left(x_{3}^{\xi}\right) & =r q_{1} f\left(x_{1}^{\xi}\right)+q_{2} f\left(x_{2}^{\xi}\right)+(1-r) q_{1} f\left(x_{1}^{\xi}\right)+q_{3} f\left(x_{3}^{\xi}\right) \\
& \in \bigcup_{z \in Z} \mathrm{LC}(f, 2, z)+\bigcup_{z \in Z} \operatorname{LC}(f, 2, z) .
\end{aligned}
$$

From $(\bullet)$ we see that if $\operatorname{Lin}_{\mathbb{Q}}\left(x_{1}^{\xi}, x_{2}^{\xi}, x_{3}^{\xi}\right) \cap \operatorname{Lin}_{\mathbb{Q}}(X) \neq\{0\}$ holds for $\mathfrak{c}$-many $\xi$ then the set $Z$ satisfies the condition $\left|\bigcup_{z \in Z} \mathrm{LC}(f, 2, z)\right|=\mathfrak{c}$. Obviously $Z \in[\mathbb{R}]^{<\mathfrak{c}}$. Thus, case (a) holds.

Summarizing the above discussion, we just need to consider a situation when $\operatorname{dim}\left(\left\{x_{1}^{\xi}, x_{2}^{\xi}, x_{3}^{\xi}\right\}\right)=2$ and $\operatorname{Lin}_{\mathbb{Q}}\left(x_{1}^{\xi}, x_{2}^{\xi}, x_{3}^{\xi}\right) \cap \operatorname{Lin}_{\mathbb{Q}}(X)=\{0\}$ for all $\xi$. Recall that $q_{1} x_{1}^{\xi}+q_{2} x_{2}^{\xi}+q_{3} x_{3}^{\xi}=0$, where $q_{1}, q_{2}, q_{3} \in \mathbb{Q} \backslash\{0\}$. If two of $x_{1}^{\xi}, x_{2}^{\xi}, x_{3}^{\xi}$ were dependent over $\mathbb{Q}$ then we would have $\operatorname{dim}\left(\left\{x_{1}^{\xi}, x_{2}^{\xi}, x_{3}^{\xi}\right\}\right) \leq 1$. Thus, $x_{1}^{\xi}, x_{2}^{\xi}, x_{3}^{\xi}$ are pairwise independent. Now it is easy to see that case (b) holds.

Lemma 3.2.7 Let $X \in[\mathbb{R}]^{<\mathfrak{c}}, x \notin X$, and $y \in \mathbb{R}$. Suppose also that $h, g: X \rightarrow \mathbb{R}$ are functions linearly independent over $\mathbb{Q}$. Then there exist extensions $h^{\prime}, g^{\prime}$ of $h$ and $g$ onto $X \cup\{x\}$ such that $h^{\prime}$ and $g^{\prime}$ are linearly independent over $\mathbb{Q}$ and $h^{\prime}(x)+g^{\prime}(x)=y$.

Proof. Choose $h^{\prime}(x) \in \mathbb{R} \backslash \operatorname{Lin}_{\mathbb{Q}}(h[X] \cup g[X] \cup\{y\})$. This choice is possible since $\left|\operatorname{Lin}_{\mathbb{Q}}(h[X] \cup g[X] \cup\{y\})\right|<\mathfrak{c}$. Then define $g^{\prime}(x)=y-h^{\prime}(x)$. It is easy to see that $h^{\prime}=h \cup\left\{\left\langle x, h^{\prime}(x)\right\rangle\right\}$ and $g^{\prime}=g \cup\left\{\left\langle x, g^{\prime}(x)\right\rangle\right\}$ are the desired extensions. 
Proof of Theorem 3.2.4. Let us start with fixing a function $f \in \mathbb{R}^{\mathbb{R}}$ and enumerations $\left\{x_{\xi}: \xi<\mathfrak{c}\right\},\left\{v_{\xi}: \xi<\mathfrak{c}\right\}$ of $\mathbb{R}$ and $\{0\} \times \mathbb{R}$, respectively. We will construct functions $h, g: \mathbb{R} \rightarrow \mathbb{R}$ which are linearly independent over $\mathbb{Q}$ and satisfy the property that $h+g=f$ and $\{0\} \times \mathbb{R} \subseteq \operatorname{Lin}_{\mathbb{Q}}(h) \cap \operatorname{Lin}_{\mathbb{Q}}(g)$.

First, let us argue that this is enough to prove the theorem. What we have to show is that $\operatorname{Lin}_{\mathbb{Q}}(h)=\operatorname{Lin}_{\mathbb{Q}}(g)=\mathbb{R}^{2}$. To see $\operatorname{Lin}_{\mathbb{Q}}(h)=\mathbb{R}^{2}$ note that

$\forall x \in \mathbb{R}^{n} \quad \forall z \in \mathbb{R} \quad\langle x, z\rangle=\langle x, h(x)\rangle+\langle\mathbf{0}, z-h(x)\rangle \in \operatorname{Lin}_{\mathbb{Q}}(h)+\operatorname{Lin}_{\mathbb{Q}}(h)=\operatorname{Lin}_{\mathbb{Q}}(h)$.

By the same argument $\operatorname{Lin}_{\mathbb{Q}}(g)=\mathbb{R}^{2}$.

To construct the desired functions $h$ and $g$, we consider three cases. In the first case we assume that $|\mathrm{LC}(f)|<\mathfrak{c}$. If the latter fails, that is $|\mathrm{LC}(f)|=\mathfrak{c}$, then either part (a) (Case 2) or part (b) (Case 3) of Property 3.2.6 holds.

Case 1: $|\mathrm{LC}(f)|=\kappa<\mathfrak{c}$.

Let $c \in \mathrm{LC}(f)$ and $A \subseteq \mathbb{R}$ be a linearly independent set over $\mathbb{Q}$ such that $\left|\operatorname{Lin}_{\mathbb{Q}}(A)\right|$ $=|\mathrm{LC}(f)|$ and $f(-a)+f(a) \equiv c=$ const for all $a \in A$. Such a set can be found since $|\mathrm{LC}(f)|<\mathfrak{c}$ and $f(x)+f(-x) \in \mathrm{LC}(f)$ for every $x \in \mathbb{R}$. Put $B=(-A) \cup A$.

First, we will construct functions $h, g: B \rightarrow \mathbb{R}$ linearly independent over $\mathbb{Q}$ for which $h+g \subseteq f$ and

$$
\{0\} \times \operatorname{LC}(f) \subseteq \operatorname{Lin}_{\mathbb{Q}}(h) \cap(\{0\} \times \mathbb{R})=\operatorname{Lin}_{\mathbb{Q}}(g) \cap(\{0\} \times \mathbb{R})
$$

To accomplish this let us fix enumerations $\left\{a_{\xi}: \xi<\kappa\right\}$ of $A$ and $\left\{m_{\xi}: \xi<\kappa\right\}$ of a linear basis of $\operatorname{LC}(f)$ over $\mathbb{Q}$. We may assume that $m_{0}=c$ if $c \neq 0$. The construction of $h$ and $g$ is by induction. At every step $\alpha<\kappa$ we will define $h$ and $g$ on $\left\{-a_{\alpha}, a_{\alpha}\right\}$, assuring that 
(a) $h\left|A_{\alpha}, g\right| A_{\alpha}$ are linearly independent and $(h+g) \mid A_{\alpha} \subseteq f$,

(b) $\left\langle 0, m_{\alpha}\right\rangle \in \operatorname{Lin}_{\mathbb{Q}}\left(h \mid A_{\alpha}\right) \cap(\{0\} \times \mathbb{R})=\operatorname{Lin}_{\mathbb{Q}}\left(g \mid A_{\alpha}\right) \cap(\{0\} \times \mathbb{R})$,

where $A_{\alpha}=\left\{i a_{\xi}: \xi \leq \alpha, i=-1,1\right\}$.

For $\alpha=0$ and $x= \pm a_{0}$ put $h(x)=\frac{1}{4} m_{0}$ and $g(x)=f(x)-h(x)$. Observe that $g\left(-a_{0}\right)+g\left(a_{0}\right)=\left[f\left(-a_{0}\right)+f\left(a_{0}\right)\right]-\left[h\left(-a_{0}\right)+h\left(a_{0}\right)\right] \in\left\{-\frac{1}{2} m_{0}, \frac{1}{2} m_{0}\right\}$. This holds because $f\left(-a_{0}\right)+f\left(a_{0}\right)=c$ and $m_{0}=c$ if $c \neq 0$. Thus $\langle 0, c\rangle,\left\langle 0, m_{0}\right\rangle \in$ $\operatorname{Lin}_{\mathbb{Q}}\left(h \mid A_{0}\right) \cap \operatorname{Lin}_{\mathbb{Q}}\left(g \mid A_{0}\right)$. It is easily seen that $h \mid A_{0}$ and $g \mid A_{0}$ satisfy (a) and (b).

Now suppose that $h$ and $g$ are defined on $A_{<\alpha}=\bigcup_{\xi<\alpha} A_{\xi}(\alpha<\kappa)$ and they satisfy the conditions (a) and (b) for all $\xi<\alpha$. We will extend $h$ and $g$ onto $A_{\alpha}$ preserving the desired properties.

We may assume that $\left\langle 0, m_{\alpha}\right\rangle \notin \operatorname{Lin}_{\mathbb{Q}}\left(h \mid A_{<\alpha}\right) \cup \operatorname{Lin}_{\mathbb{Q}}\left(g \mid A_{<\alpha}\right)$. (Otherwise we could extend $h$ and $g$ using Lemma 3.2.7 preserving the condition (a).) Put $h(x)=\frac{1}{2} m_{\alpha}$ and $g(x)=f(x)-h(x)$ for $x \in\left\{-a_{\alpha}, a_{\alpha}\right\}$. We claim that (a) and (b) are satisfied. Obviously, $(h+g) \mid A_{\alpha} \subseteq f$. To see the linear independence of $h \mid A_{\alpha}$ and $g \mid A_{\alpha}$ first note that, based on the inductive assumption, $h \mid A_{<\alpha}$ and $g \mid A_{<\alpha}$ are linearly independent. Next suppose that

$$
p\left\langle-a_{\alpha}, h\left(-a_{\alpha}\right)\right\rangle+q\left\langle a_{\alpha}, h\left(a_{\alpha}\right)\right\rangle=v \text { for some } p, q \in \mathbb{Q} \text { and } v \in \operatorname{Lin}_{\mathbb{Q}}\left(h \mid A_{<\alpha}\right) .
$$

Since $a_{\alpha} \notin \operatorname{Lin}_{\mathbb{Q}}\left(A_{<\alpha}\right)$ we conclude that $p=q$. Therefore we have

$$
p\left\langle-a_{\alpha}, h\left(-a_{\alpha}\right)\right\rangle+q\left\langle a_{\alpha}, h\left(a_{\alpha}\right)\right\rangle=p\left\langle 0, h\left(-a_{\alpha}\right)+h\left(a_{\alpha}\right)\right\rangle=p\left\langle 0, m_{\alpha}\right\rangle=v .
$$

But we assumed that $\left\langle 0, m_{\alpha}\right\rangle \notin \operatorname{Lin}_{\mathbb{Q}}\left(h \mid A_{<\alpha}\right) \cup \operatorname{Lin}_{\mathbb{Q}}\left(g \mid A_{<\alpha}\right)$, so $p=0$ and $v=\mathbf{0}$. This shows linear independence of $h \mid A_{\alpha}$. Very similar argument works for $g \mid A_{\alpha}$ : just 
notice that $g\left(-a_{\alpha}\right)+g\left(a_{\alpha}\right)=\left[f\left(-a_{\alpha}\right)+f\left(a_{\alpha}\right)\right]-\left[h\left(-a_{\alpha}\right)+h\left(a_{\alpha}\right)\right]=c-m_{\alpha}$ and recall that $\langle 0, c\rangle \in \operatorname{Lin}_{\mathbb{Q}}\left(g \mid A_{0}\right) \subseteq \operatorname{Lin}_{\mathbb{Q}}\left(g \mid A_{<\alpha}\right)$.

Now we show that (b) is also satisfied. From what has already been proved, we conclude that $\left\langle 0, m_{\alpha}\right\rangle \in \operatorname{Lin}_{\mathbb{Q}}\left(h \mid A_{\alpha}\right) \cup \operatorname{Lin}_{\mathbb{Q}}\left(g \mid A_{\alpha}\right)$.

Thus, what remains to prove is the equality part of (b). (The following argument is also needed in the case when Lemma 3.2.7 was used to define $h$ and $g$ on $\left\{-a_{\alpha}, a_{\alpha}\right\}$.) It follows from the fact that $\langle 0, y\rangle \in \operatorname{Lin}_{\mathbb{Q}}\left(h \mid A_{\alpha}\right)$ provided there exist $p_{i} \in \mathbb{Q}$ and $a_{i} \in A_{\alpha}, i \leq n$ such that

$$
\begin{aligned}
\langle 0, y\rangle & =\sum_{1}^{n} p_{i}\left[\left\langle-a_{i}, h\left(-a_{i}\right)\right\rangle+\left\langle a_{i}, h\left(a_{i}\right)\right\rangle\right] \\
& =\sum_{1}^{n} p_{i}\left\langle 0, h\left(-a_{i}\right)+h\left(a_{i}\right)\right\rangle \\
& =\sum_{1}^{n} p_{i}\left\langle 0, f\left(-a_{i}\right)+f\left(a_{i}\right)\right\rangle-p_{i}\left\langle 0, g\left(-a_{i}\right)+g\left(a_{i}\right)\right\rangle \\
& =\sum_{1}^{n} p_{i}\langle 0, c\rangle-\sum_{1}^{n} p_{i}\left[\left\langle-a_{i}, g\left(-a_{i}\right)\right\rangle+\left\langle a_{i}, g\left(a_{i}\right)\right\rangle\right] \\
& \in \operatorname{Lin}_{\mathbb{Q}}\left(g \mid A_{\alpha}\right) .
\end{aligned}
$$

This completes the inductive definition of $h$ and $g$. Note that (3.4) implies that any extensions $h^{\prime}, g^{\prime}$ of $h$ and $g$, with $h^{\prime}+g^{\prime} \subseteq f$, satisfy also

$$
\{0\} \times \operatorname{LC}(f) \subseteq \operatorname{Lin}_{\mathbb{Q}}\left(h^{\prime}\right) \cap(\{0\} \times \mathbb{R})=\operatorname{Lin}_{\mathbb{Q}}\left(g^{\prime}\right) \cap(\{0\} \times \mathbb{R}) .
$$

To see this choose $\langle 0, y\rangle \in \operatorname{Lin}_{\mathbb{Q}}\left(h^{\prime}\right) \cap(\{0\} \times \mathbb{R})$. So for some $p_{i} \in \mathbb{Q}$ and $x_{i} \in \mathbb{R}$ we have $\langle 0, y\rangle=\sum_{1}^{n} p_{i}\left\langle x_{i}, h^{\prime}\left(x_{i}\right)\right\rangle=\sum_{1}^{n} p_{i}\left\langle x_{i}, f\left(x_{i}\right)\right\rangle-\sum_{1}^{n} p_{i}\left\langle x_{i}, g^{\prime}\left(x_{i}\right)\right\rangle \in \operatorname{Lin}_{\mathbb{Q}}\left(g^{\prime}\right) \cap(\{0\} \times \mathbb{R})$. The latter holds because $\sum_{1}^{n} p_{i} x_{i}=0$ and consequently $\sum_{1}^{n} p_{i} f\left(x_{i}\right) \in \mathrm{LC}(f)$. This ends the proof of (3.5). 
Next we extend $h$ and $g$ onto $\mathbb{R}=\left\{x_{\xi}: \xi<\mathfrak{c}\right\}$, preserving the linear independence and the property that at the step $\xi$ of the inductive definition we assure that $x_{\xi} \in$ $\operatorname{dom}\left(h_{\xi}\right)=\operatorname{dom}\left(g_{\xi}\right)$ and $v_{\xi} \in\{0\} \times \operatorname{Lin}_{\mathbb{Q}}\left(h_{\xi}\right)=\{0\} \times \operatorname{Lin}_{\mathbb{Q}}\left(g_{\xi}\right)$, where $h_{\xi}$ and $g_{\xi}$ denote the extensions obtained in the step $\xi$.

Let $\beta<\mathfrak{c}$. Assume that $v_{\beta} \notin\{0\} \times \operatorname{Lin}_{\mathbb{Q}}\left(\bigcup_{\xi<\beta} h_{\xi}\right)=\{0\} \times \operatorname{Lin}_{\mathbb{Q}}\left(\bigcup_{\xi<\beta} g_{\xi}\right)$. Choose an $a \in \mathbb{R} \backslash \operatorname{Lin}_{\mathbb{Q}}\left(\operatorname{dom}\left(\bigcup_{\xi<\beta} h_{\xi}\right)\right)$ and define $h(x)$ in such a way that $\langle 0, h(x)\rangle=\frac{1}{2} v_{\beta}$ for $x \in\{-a, a\}$. Put also $g(x)=f(x)-h(x)$. Since $f(-a)+f(a) \in \operatorname{LC}(f),(3.4)$ implies that $v_{\beta} \in \operatorname{Lin}_{\mathbb{Q}}(h) \cap \operatorname{Lin}_{\mathbb{Q}}(g)$. What remains to show is that $h, g$ are still linearly independent. But this follows from (3.5) and almost the same argument which is used to show linear independence of $h \mid A_{\alpha}$ and $g \mid A_{\alpha}$ in the previous part of the proof. (Replace $a_{\alpha}, h \mid A_{\alpha}$, and $g \mid A_{\alpha}$ by $a, \bigcup_{\xi<\beta} h_{\xi}$, and $\bigcup_{\xi<\beta} g_{\xi}$, respectively.)

To finish the step $\beta$ of the inductive definition we need to make sure that $h$ and $g$ are defined at $x_{\beta}$. If $x_{\beta} \notin \operatorname{dom}(h)=\operatorname{dom}(g)$ then we can use Lemma 3.2.7 to define these functions at $x_{\beta}$, preserving all the required properties.

This ends the construction in Case 1.

Case 2: Property 3.2.6 (a) holds.

Let $Z \in[\mathbb{R}]^{<\mathfrak{c}}$ be a set satisfying

$$
\left|\bigcup_{z \in Z} \operatorname{LC}(f, 2, z)\right|=\mathfrak{c} .
$$

We start with defining functions $h, g: Z \rightarrow \mathbb{R}$ which are linearly independent over $\mathbb{Q}$ and whose sum is contained in $f$ (i.e., $h+g \subseteq f$.) It can be easily done by using Lemma 3.2.7.

We will extend $h$ and $g$ onto $\mathbb{R}$ by induction. Let $\beta<\mathfrak{c}$. Assume that $h$ and $g$ are linearly independent, $h+g \subseteq f,\left\{x_{\xi}: \xi<\beta\right\} \subseteq D_{\beta}=\operatorname{dom}(h)=\operatorname{dom}(g)$, 
$\left\{v_{\xi}: \xi<\beta\right\} \subseteq \operatorname{Lin}_{\mathbb{Q}}(h) \cap \operatorname{Lin}_{\mathbb{Q}}(g)$, and $v_{\beta} \notin \operatorname{Lin}_{\mathbb{Q}}(h)$. The property of the set $Z$ implies the existence of a $z \in Z$ satisfying $|\mathrm{LC}(f, 2, z)|>\max (|h|, \omega)=\max (|g|, \omega)$. Thus, an easy cardinal argument shows that we can find $z_{1}, z_{2} \in \mathbb{R} \backslash \operatorname{Lin}_{\mathbb{Q}}\left(D_{\beta}\right)$ and $p \in \mathbb{Q} \backslash\{0\}$ which satisfy

$$
z_{1}+p z_{2}=z \text { and }\left\langle z, f\left(z_{1}\right)+p f\left(z_{2}\right)\right\rangle \notin \operatorname{Lin}_{\mathbb{Q}}\left(g \cup\left\{\langle 0, h(z)\rangle, v_{\beta}\right\}\right) .
$$

Define the values of $h$ at $z_{1}$ and $z_{2}$ so that

$$
\left\langle z_{1}, h\left(z_{1}\right)\right\rangle+p\left\langle z_{2}, h\left(z_{2}\right)\right\rangle=\left\langle z, h\left(z_{1}\right)+p h\left(z_{2}\right)\right\rangle=v_{\beta}+\langle z, h(z)\rangle .
$$

Observe that then $v_{\beta}=\left[v_{\beta}+\langle z, h(z)\rangle\right]-\langle z, h(z)\rangle \in \operatorname{Lin}_{\mathbb{Q}}(h)$.

Now we argue that $h$ and $g$ still linearly independent. To see linear independence of $h$ suppose that for some $q, r \in \mathbb{Q}$ (not both equal 0) we have

$$
q\left\langle z_{1}, h\left(z_{1}\right)\right\rangle+r\left\langle z_{2}, h\left(z_{2}\right)\right\rangle=\left\langle q z_{1}+r z_{2}, q h\left(z_{1}\right)+r h\left(z_{2}\right)\right\rangle \in \operatorname{Lin}_{\mathbb{Q}}\left(h \mid D_{\beta}\right) .
$$

Since $z_{1}, z_{2} \notin \operatorname{Lin}_{\mathbb{Q}}\left(D_{\beta}\right)$ and $z_{1}+p z_{2}=z \in Z \subseteq \operatorname{Lin}_{\mathbb{Q}}\left(D_{\beta}\right)$ we conclude that $\langle q, r\rangle$ and $\langle 1, p\rangle$ are linearly dependent. So we may assume that $\langle q, r\rangle=\langle 1, p\rangle$. Consequently, $v_{\beta}+\langle z, h(z)\rangle=\left\langle z, h\left(z_{1}\right)+p h\left(z_{2}\right)\right\rangle \in \operatorname{Lin}_{\mathbb{Q}}\left(h \mid D_{\beta}\right)$. This contradicts the assumption $v_{\beta} \notin \operatorname{Lin}_{\mathbb{Q}}\left(h \mid D_{\beta}\right)$. Hence, $h$ is linearly independent.

Based on the above argument, we see that linear independence of $g$ will follow from $\left\langle z, g\left(z_{1}\right)+p g\left(z_{2}\right)\right\rangle \notin \operatorname{Lin}_{\mathbb{Q}}\left(g \mid D_{\beta}\right)$. But this holds since (3.6) imply

$$
\begin{aligned}
\left\langle z, g\left(z_{1}\right)+p g\left(z_{2}\right)\right\rangle & = \\
\left\langle z, f\left(z_{1}\right)+p f\left(z_{2}\right)-\left[h\left(z_{1}\right)+p h\left(z_{2}\right)\right]\right\rangle & = \\
\left\langle z, f\left(z_{1}\right)+p f\left(z_{2}\right)\right\rangle-\langle 0, h(z)\rangle-v_{\beta} & \notin \operatorname{Lin}_{\mathbb{Q}}\left(g \mid D_{\beta}\right) .
\end{aligned}
$$


To assure that $v_{\beta} \in \operatorname{Lin}_{\mathbb{Q}}(g)$ we repeat the same procedure as above for the function $g$. Finally, if $x_{\beta} \notin \operatorname{dom}(h)=\operatorname{dom}(g)$ then we use Lemma 3.2.7 to define the functions at $x_{\beta}$.

This ends the construction in Case 2 .

Case 3: Property 3.2.6 (b) holds.

The inductive construction of functions $h$ and $g$ is somewhat similar to the one from the previous case. So assume that $\beta<\mathfrak{c}$ and the construction has been carried out for all $\xi<\beta$. If $v_{\beta} \notin \operatorname{Lin}_{\mathbb{Q}}(h)$ then let $X=\operatorname{dom}(h)=\operatorname{dom}(g)$ and $Y \in[\mathbb{R}]^{<\mathfrak{c}}$ be such a set that $\operatorname{Lin}_{\mathbb{Q}}\left(g \cup\left\{v_{\beta}\right\}\right) \subseteq \mathbb{R} \times Y$. By Property 3.2.6 (b), there exist $p_{1}, p_{2}, p_{3} \in \mathbb{Q} \backslash\{0\}$ and pairwise independent $x_{1}, x_{2}, x_{3} \in \mathbb{R}$ such that $\sum_{1}^{3} p_{i} x_{i}=0$, $\operatorname{Lin}_{\mathbb{Q}}\left(x_{1}, x_{2}, x_{3}\right) \cap \operatorname{Lin}_{\mathbb{Q}}(X)=\{0\}$, and $\sum_{1}^{3} p_{i} f\left(x_{i}\right) \notin Y$.

We extend $h$ and $g$ onto $\left\{x_{1}, x_{2}, x_{3}\right\}$. Choose $h\left(x_{1}\right), h\left(x_{2}\right), h\left(x_{3}\right) \in \mathbb{R}$ in such a way that

$$
\sum_{1}^{3} p_{i}\left\langle x_{i}, h\left(x_{i}\right)\right\rangle=\left\langle 0, \sum_{1}^{3} p_{i} h\left(x_{i}\right)\right\rangle=v_{\beta} .
$$

Then put $g\left(x_{i}\right)=f\left(x_{i}\right)-h\left(x_{i}\right)$ for $i \leq 3$. Obviously $v_{\beta} \in \operatorname{Lin}_{\mathbb{Q}}(h)$ and $h+g \subseteq f$. We claim that linear independence of $h$ and $g$ is also preserved.

To show this claim notice first, that if $\sum_{1}^{3} p_{i}^{\prime} x_{i} \in \operatorname{Lin}_{\mathbb{Q}}(X)$ for some $p_{1}^{\prime}, p_{2}^{\prime}, p_{3}^{\prime} \in \mathbb{Q}$ then $\sum_{1}^{3} p_{i}^{\prime} x_{i}=0$. Pairwise independence of $x_{1}, x_{2}, x_{3}$ implies that $\sum_{1}^{3} p_{i}^{\prime} x_{i}=0$ holds only for $\left\langle p_{1}^{\prime}, p_{2}^{\prime}, p_{3}^{\prime}\right\rangle \in \operatorname{Lin}_{\mathbb{Q}}\left(\left\langle p_{1}, p_{2}, p_{3}\right\rangle\right)$. Thus, our claim holds if $\sum_{1}^{3} p_{i}\left\langle x_{i}, h\left(x_{i}\right)\right\rangle \notin$ $\operatorname{Lin}_{\mathbb{Q}}(h \mid X)$ and $\sum_{1}^{3} p_{i}\left\langle x_{i}, g\left(x_{i}\right)\right\rangle \notin \operatorname{Lin}_{\mathbb{Q}}(g \mid X)$. But these two conditions follow from

- $\sum_{1}^{3} p_{i}\left\langle x_{i}, h\left(x_{i}\right)\right\rangle=v_{\beta} \notin \operatorname{Lin}_{\mathbb{Q}}(h \mid X)$ and

- $\sum_{1}^{3} p_{i}\left\langle x_{i}, g\left(x_{i}\right)\right\rangle=\sum_{1}^{3} p_{i}\left\langle x_{i}, f\left(x_{i}\right)-h\left(x_{i}\right)\right\rangle=\left\langle 0, \sum_{1}^{3} p_{i} f\left(x_{i}\right)\right\rangle-v_{\beta} \notin \operatorname{Lin}_{\mathbb{Q}}(g \mid X)$ ( " $\notin$ " part holds because $\operatorname{Lin}_{\mathbb{Q}}\left((g \mid X) \cup\left\{v_{\beta}\right\}\right) \subseteq \mathbb{R} \times Y$ and $\sum_{1}^{3} p_{i} f\left(x_{i}\right) \notin Y$.) 
To assure that $v_{\beta} \in \operatorname{Lin}_{\mathbb{Q}}(g)$ we repeat the same steps as above for the function $g$ and then, if necessary, define $h$ and $g$ at $x_{\beta}$ using Lemma 3.2.7.

This ends the construction in Case 3. 


\section{Chapter 4}

\section{Generalized continuity versus additivity}

\subsection{Introduction}

In this chapter we investigate the function Add for pairs of classes that are not considered in the previous part of this work. More precisely, in Section 4.2 we describe $\operatorname{Add}\left(\mathcal{F}_{1}, \mathcal{F}_{2}\right)$ in the case when both $\mathcal{F}_{1}$ and $\mathcal{F}_{2}$ are Darboux-like. Section 4.3 deals with the situation when one family is Darboux-like and the other one is the family of additive or Hamel functions.

Before we proceed to the next sections we recall some definitions and cite some theorems. Let $h \in$ Ext. We say that a set $G \subset \mathbb{R}$ is $h$-negligible provided $f \in$ Ext for every function $f: \mathbb{R} \rightarrow \mathbb{R}$ for which $f=h$ on a set $\mathbb{R} \backslash G$. For a cardinal number $\kappa \leq \mathfrak{c}$, a function $f: \mathbb{R} \rightarrow \mathbb{R}$ is called $\kappa$-strongly Darboux if $f^{-1}(y)$ is $\kappa$-dense for every $y \in \mathbb{R}$. If $\kappa=\omega$ then we simply say that $f$ is strongly Darboux. We denote the family of all $\kappa$-strongly Darboux functions by $\mathrm{D}(\kappa)$. It is obvious from the definition that

$$
\mathrm{D}(\lambda) \subseteq \mathrm{D}(\kappa) \text { for all cardinals } \kappa \leq \lambda \leq \mathfrak{c}
$$


We also introduce the family $\mathrm{D}(\mathrm{P})$ of perfectly Darboux functions as the class of all functions $f: \mathbb{R} \rightarrow \mathbb{R}$ such that $Q \cap f^{-1}(y) \neq \emptyset$ for every perfect set $Q \subseteq \mathbb{R}$ and $y \in \mathbb{R}$. In other words, a function $f$ is perfectly Darboux if $f^{-1}(y)$ is a Bernstein set for every $y \in \mathbb{R}$. Notice that $\mathrm{D}(\mathrm{P}) \subseteq \mathrm{D}(\kappa)$ for every $\kappa \leq \mathfrak{c}$.

The following theorem is proved in [5].

Theorem 4.1.1. $\mathrm{A}(\mathrm{AC})=\mathrm{A}(\mathrm{D})=\mathrm{A}\left(\mathrm{D}\left(\omega_{1}\right)\right)$.

Using a similar technique as in the case of the above theorem, we will prove the following proposition.

Proposition 4.1.2 Let $\mathcal{F} \in\{\mathrm{AD}, \mathrm{HF}, \operatorname{Ext}\}$. Then $\operatorname{Add}(\mathcal{F}, \mathrm{AC})=\operatorname{Add}(\mathcal{F}, \mathrm{D})$.

The proof of Proposition 4.1.2 requires the use of other lemmas and a proposition.

Lemma 4.1.3 Let $X$ be any set of cardinality continuum and assume that $F \subseteq \mathbb{R}^{X}$ satisfies the condition $|F|<\mathrm{A}(\mathrm{D})$. There exists a $g: X \rightarrow \mathbb{R}$ such that $(g+f)^{-1}(y) \neq$ $\emptyset$ for each $y \in \mathbb{R}$.

Proof. Let $b: \mathbb{R} \rightarrow X$ be a bijection. By Theorem 4.1.1 and monotonicity of $\mathrm{A}$ we have that $\mathrm{A}(\mathrm{D})=\mathrm{A}(\mathrm{D}(\omega))$. Hence we can find a $g^{\prime}: \mathbb{R} \rightarrow \mathbb{R}$ satisfying the property that $g^{\prime}+(f \circ b) \in \mathrm{D}(\omega)$ for each $f \in F$. Put $g=g^{\prime} \circ b^{-1}$. Clearly, $g$ is the desired function.

Lemma 4.1.4 Let $X \subseteq \mathbb{R}$ be a set linearly independent over $\mathbb{Q}$. Then every function $f: X \rightarrow \mathbb{R}$ can be extended onto $\mathbb{R}$ to a Hamel function. 
Proof. Let $g \in \mathbb{R}^{\mathbb{R}}$ be a Hamel function. Define $g^{\prime}: \mathbb{R} \rightarrow \mathbb{R}$ to be an additive extension of $f-(g \mid X)$. Notice that $g+g^{\prime} \in$ HF by Fact 3.2.1 (i). Since $\left(g+g^{\prime}\right) \mid X=f$ we conclude that $g+g^{\prime}$ is a Hamel function extending $f$.

Proposition 4.1.5 $\mathrm{A}(\mathrm{D})=\mathrm{A}(\mathrm{D}(\mathrm{P}))$.

ProOF. Fix a family $F \subseteq \mathbb{R}^{\mathbb{R}}$ of cardinality less than A(D). Next, let $\left\{B_{\xi}: \xi<\mathfrak{c}\right\}$ and $\left\{P_{\xi}: \xi<\mathfrak{c}\right\}$ be a family of pairwise disjoint Bernstein sets and an enumeration of all perfect subsets of $\mathbb{R}$, respectively. We define the sequence $\left\langle A_{\xi}: \xi<\mathfrak{c}\right\rangle$ by $A_{\xi}=B_{\xi} \cap P_{\xi}$. Obviously, the sets $A_{\xi}$ are pairwise disjoint and each one of them has cardinality $\mathfrak{c}$. Applying Lemma 4.1 .3 for every $\xi<\mathfrak{c}$ separately, we get a sequence of functions $\left\langle g_{\xi}: A_{\xi} \rightarrow \mathbb{R} \mid \xi<\mathfrak{c}\right\rangle$ such that for every $\xi<\mathfrak{c}$ the following holds

$$
\forall f \in F \quad \forall y \in \mathbb{R} \quad\left(g_{\xi}+f\right)^{-1}(y) \neq \emptyset .
$$

Now, if $g \in \mathbb{R}^{\mathbb{R}}$ is any extension of $\bigcup_{\xi<\mathfrak{c}} g_{\xi}$ onto $\mathbb{R}$ then $g+F \subseteq \mathrm{D}(\mathrm{P})$.

Proof of Proposition 4.1.2. First we show that

$(* *) \operatorname{Add}\left(\mathcal{F}, \mathcal{F}_{0}\right)>\mathfrak{c}$ for $\mathcal{F}_{0} \in\left\{\mathrm{AC}, \mathrm{D}\left(\omega_{1}\right)\right\}$

Let us fix a family $F \subseteq \mathbb{R}^{\mathbb{R}}$ with cardinality $\mathfrak{c}$. To prove the case $\mathcal{F} \in\{\mathrm{AD}, \mathrm{HF}\}$ consider a c-dense Hamel basis $H$. There exists a partition $\left\{B_{f}: f \in F\right\}$ of $H$ into $\mathfrak{c}$-dense sets. Since the projection of every blocking set in $\mathbb{R}^{2}$ contains an interval, we can find, for every $f \in F$, a partial function $g_{f}: B_{f} \rightarrow \mathbb{R}$ such that $g_{f}+f$ intersects every blocking set in at least $\omega_{1}$ points. Thus every extension of $g_{f}+f$ onto $\mathbb{R}$ is almost continuous and $\omega_{1}$-strongly Darboux. If $g \in \mathbb{R}^{\mathbb{R}}$ is any function containing 
$\bigcup_{f \in F} g_{f}$ then $g+F \subseteq \mathrm{AC} \cap \mathrm{D}\left(\omega_{1}\right)$. In particular, we can choose $g$ to be an additive or Hamel function (see Lemma 4.1.4.) Hence $\operatorname{Add}\left(\mathcal{F}, \mathcal{F}_{0}\right)>\mathfrak{c}$ for $\mathcal{F}_{0} \in\left\{\operatorname{AC}, \mathrm{D}\left(\omega_{1}\right)\right\}$.

Now consider the case $\mathcal{F}=$ Ext. If $\mathcal{F}_{0}=\mathrm{AC}$ then we have the inequality $\operatorname{Add}($ Ext, $\mathrm{AC}) \geq \operatorname{Add}($ Ext, Ext $)=\mathrm{A}($ Ext $)=\mathfrak{c}^{+}>\mathfrak{c}$ which follows from Proposition 1.2.1 (2) \& (5). Now, let us focus on the case $\mathcal{F}_{0}=\mathrm{D}\left(\omega_{1}\right)$. Let $Q \subseteq \mathbb{R}$ be a $\mathfrak{c}$-dense meager $F_{\sigma}$-set. Then, according to [4, Proposition 4.3], there exists an extendable function $f: \mathbb{R} \rightarrow \mathbb{R}$ such that the set $\mathbb{R} \backslash Q$ is $f$-negligible. Since $|F|<\mathrm{A}(\mathrm{D})=\mathrm{A}(\mathrm{D}(\mathrm{P}))$, there exists a function $h \in \mathbb{R}^{\mathbb{R}}$ such that $h+F \subseteq \mathrm{D}(\mathrm{P})$. Notice here that any perfectly Darboux function modified on a meager set is in $\mathrm{D}\left(\omega_{1}\right)$. This implies that the function $g=f|Q \cup h|(\mathbb{R} \backslash Q)$ shifts $F$ into $\mathrm{D}\left(\omega_{1}\right) \subseteq \mathrm{D}$. Since $Q \subseteq[f=g]$ we have that $g \in$ Ext. Observe also that $F$ could be any family with $|F|<\mathrm{A}(\mathrm{D})=\mathrm{A}(\mathrm{D}(\mathrm{P}))$. So we actually proved that

$$
\operatorname{Add}(\operatorname{Ext}, \mathrm{D}) \geq \operatorname{Add}\left(\operatorname{Ext}, \mathrm{D}\left(\omega_{1}\right)\right) \geq \mathrm{A}(\mathrm{D}) \text {. }
$$

This finishes the proof of $(* *)$.

Now the argument follows the schema of the proof of Theorem 4.1.1. ${ }^{1}$ We start with proving the equality $\operatorname{Add}(\mathcal{F}, \mathrm{D})=\operatorname{Add}\left(\mathcal{F}, \mathrm{D}\left(\omega_{1}\right)\right)$. Obviously, $\operatorname{Add}(\mathcal{F}, \mathrm{D}) \geq$ $\operatorname{Add}\left(\mathcal{F}, \mathrm{D}\left(\omega_{1}\right)\right)$. To justify the other inequality let $\kappa=\operatorname{Add}\left(\mathcal{F}, \mathrm{D}\left(\omega_{1}\right)\right)$. By $(* *)$ we get that $\kappa>\mathfrak{c}$. We will show that $\kappa \geq \operatorname{Add}(\mathcal{F}, D)$.

Consider a family $G \subseteq \mathbb{R}^{\mathbb{R}}$ of cardinality $\kappa$ witnessing $\kappa=\operatorname{Add}\left(\mathcal{F}, \mathrm{D}\left(\omega_{1}\right)\right)$. We define a new family $G^{*}=\left\{h \in \mathbb{R}^{\mathbb{R}}: \exists g \in G h={ }^{*} g\right\}$, where $h={ }^{*} f$ if and only if $|\{x: h(x) \neq f(x)\}| \leq \omega$. Notice here that $\left|G^{*}\right|=\kappa$. This is so because $\kappa>\mathfrak{c}$ and for every $f \in \mathbb{R}^{\mathbb{R}}$ the set $\left\{h \in \mathbb{R}^{\mathbb{R}}: h=^{*} f\right\}$ has cardinality $\mathfrak{c}$. We claim that

\footnotetext{
${ }^{1}$ For reader's convenience, we include this slight modification of the proof from [5] in this paper.
} 
$G^{*}$ witnesses $\kappa \geq \operatorname{Add}(\mathcal{F}, \mathrm{D})$. Indeed, let $f \in \mathcal{F}$. Then, by the choice of $G$, there exists a $g \in G$ satisfying the following $f+g \notin \mathrm{D}\left(\omega_{1}\right)$. This implies the existence of a non-trivial closed interval $I$ and $y \in \mathbb{R}$ for which $\left|I \cap(f+g)^{-1}(y)\right| \leq \omega$. By modification of $g$ on a countable set, we get a function $g^{*} \in G^{*}$ with the property that $\left(f+g^{*}\right)[I] \cap(-\infty, y) \neq \emptyset \neq\left(f+g^{*}\right)[I] \cap(y, \infty)$ and $y \notin\left(f+g^{*}\right)[I]$. Therefore $\left(f+g^{*}\right) \notin \mathrm{D}$. This ends the proof of the equality $\operatorname{Add}(\mathcal{F}, \mathrm{D})=\operatorname{Add}\left(\mathcal{F}, \mathrm{D}\left(\omega_{1}\right)\right)$.

What remains to show is that $\operatorname{Add}(\mathcal{F}, \mathrm{AC})=\operatorname{Add}\left(\mathcal{F}, \mathrm{D}\left(\omega_{1}\right)\right)$. The inequality $\operatorname{Add}(\mathcal{F}, \operatorname{AC}) \leq \operatorname{Add}(\mathcal{F}, \mathrm{D})=\operatorname{Add}\left(\mathcal{F}, \mathrm{D}\left(\omega_{1}\right)\right)$ is obvious, so we just need to prove that $\operatorname{Add}(\mathcal{F}, \mathrm{AC}) \geq \operatorname{Add}\left(\mathcal{F}, \mathrm{D}\left(\omega_{1}\right)\right)$. This time consider $K \subseteq \mathbb{R}^{\mathbb{R}}$ witness$\operatorname{ing} \operatorname{Add}(\mathcal{F}, \mathrm{AC})=\lambda$. We put $K^{*}=\left\{g-h_{B}: g \in K\right.$ and $B$ is a blocking set $\}$, where $h_{B} \in \mathbb{R}^{\mathbb{R}}$ is a function such that $h_{B} \mid \operatorname{dom}(B) \subseteq B$. Clearly $\left|K^{*}\right|=\lambda$ because there are only continuum many blocking sets and $\lambda>\mathfrak{c}$. Let $f \in \mathcal{F}$. Then, by the choice of $K$, there exist a $g \in K$ and a blocking set $B$ such that $(f+g) \cap B=\emptyset$. In particular,

$$
\left[f+\left(g-h_{B}\right)\right] \cap\left(B-h_{B}\right)=[(f+g) \cap B]-h_{B}=\emptyset,
$$

where we define $Z-h_{B}=\left\{\left(x, y-h_{B}(x)\right):(x, y) \in Z\right\}$ for any $Z \subseteq \mathbb{R}^{2}$. From the definition of $h_{B}$ we have $\operatorname{dom}(B) \times\{0\} \subseteq\left(B-h_{B}\right)$. Thus $f+\left(g-h_{B}\right)$ has an empty intersection with $\operatorname{dom}(B) \times\{0\}$. This means that $f+\left(g-h_{B}\right) \notin \mathrm{D}\left(\omega_{1}\right)$, since $\operatorname{dom}(B)$ contains a non-trivial interval. But $g-h_{B} \in K^{*}$, so $K^{*}$ witnesses $\lambda \geq \operatorname{Add}\left(\mathcal{F}, \mathrm{D}\left(\omega_{1}\right)\right)$. This finishes the proof of $\operatorname{Add}(\mathcal{F}, \mathrm{AC})=\operatorname{Add}\left(\mathcal{F}, \mathrm{D}\left(\omega_{1}\right)\right)$ as well as whole Proposition 4.1.2. 


\subsection{Relations among Darboux-like families}

The following result gives the values of the function Add for pairs of Darboux-like classes.

Theorem 4.2.1. If $\mathcal{F}, \mathcal{G} \in\{$ Ext, $\mathrm{AC}, \mathrm{Conn}, \mathrm{D}, \mathrm{PC}\}$ then $\operatorname{Add}(\mathcal{F}, \mathcal{G})=\mathrm{A}(\mathcal{G})$ and $\operatorname{Add}(\mathrm{C}, \mathcal{F})=\operatorname{Add}(\mathcal{F}, \mathrm{C})=1$.

Proof. We start with proving $\operatorname{Add}(\mathrm{C}, \mathcal{F})=\operatorname{Add}(\mathcal{F}, \mathrm{C})=1$. Notice that it is enough to show the latter for $\mathcal{F}=\mathrm{PC}$ since $\operatorname{Add}(\mathrm{C}, \mathcal{F}) \leq \operatorname{Add}(\mathrm{C}, \mathrm{PC})$ and $\operatorname{Add}(\mathcal{F}, \mathrm{C}) \leq \operatorname{Add}(\mathrm{PC}, \mathrm{C})$ by Proposition 1.2.1 (1) \& (2). To see that $\operatorname{Add}(\mathrm{C}, \mathrm{PC})=$ $\operatorname{Add}(\mathrm{PC}, \mathrm{C})=1$ observe that $\mathrm{C}+\mathrm{PC}=\mathrm{PC}$. Therefore, if $f \notin \mathrm{PC}$ then there is no $g \in \mathrm{C}$ such that $g+f \in \mathrm{PC}$.

Now we prove $\operatorname{Add}(\mathcal{F}, \mathcal{G})=\mathrm{A}(\mathcal{G})$. Let us first assume that $\mathcal{G}=$ Ext. The desired conclusion follows from the inequality

$$
\mathrm{A}(\operatorname{Ext})=\operatorname{Add}\left(\mathbb{R}^{\mathbb{R}}, \operatorname{Ext}\right) \geq \operatorname{Add}(\mathcal{F}, \operatorname{Ext}) \geq \operatorname{Add}(\operatorname{Ext}, \operatorname{Ext})=\mathrm{A}(\operatorname{Ext})=\mathfrak{c}^{+},
$$

where the equality part is implied by Proposition 1.2.1 (5).

Next suppose that $\mathcal{G} \in\{\mathrm{AC}$, Conn, $\mathrm{D}\}$. By the monotonicity of Add we just need to show that $\operatorname{Add}($ Ext, $\mathcal{G})=\mathrm{A}(\mathcal{G})$. Recall that $\mathrm{A}(\mathrm{AC})=\mathrm{A}(\mathrm{Conn})=\mathrm{A}(\mathrm{D})$. and also note that, by Proposition 4.1.2 and Proposition 1.2.1 (2), Add(Ext, AC) = $\operatorname{Add}($ Ext, Conn $)=\operatorname{Add}($ Ext, D). The desired equality follows from

$$
\mathrm{A}(\mathrm{AC})=\mathrm{A}(\mathrm{Conn})=\mathrm{A}(\mathrm{D}) \geq \operatorname{Add}(\operatorname{Ext}, \mathrm{D}) \geq \mathrm{A}(\mathrm{D}),
$$

where the last inequality is shown in the proof of $(* *)$ in Proposition 4.1.2. 
What remains to prove is $\operatorname{Add}(\mathcal{F}, \mathcal{G})=\mathrm{A}(\mathcal{G})$ for $\mathcal{G}=$ PC. Again, by the monotonicity of Add, it suffices to show the latter for $\mathcal{F}=$ Ext. Let $Q \subseteq \mathbb{R}$ and $f: \mathbb{R} \rightarrow \mathbb{R}$ be as in the proof of $(* *)$ Proposition 4.1.2, i.e., $Q$ is $\mathfrak{c}$-dense meager $F_{\sigma}$-set and $f$ is an extendable function such that $\mathbb{R} \backslash Q$ is $f$-negligible. Fix a family $F \subseteq \mathbb{R}^{\mathbb{R}}$ of cardinality less than $2^{\mathfrak{c}}$. Now, a small modification in the proof of the equality $\operatorname{Add}(\mathrm{SZ}, \mathrm{PC})=2^{\mathfrak{c}}$ in Section 2.3 (the sets $B_{\langle I, p, m\rangle}$ can be chosen to be subsets of $\mathbb{R} \backslash Q$ ), gives us a function $g: \mathbb{R} \rightarrow \mathbb{R}$ which shifts $F$ into $\mathrm{PC}$ and which agrees with $f$ on the set containing $Q$. In particular, $g$ is an extendable function.

\subsection{Relations between Darboux-like and additive or Hamel functions}

In this section the cardinal function Add is investigated for pairs of classes such that one is Darboux-like and the other is either the class AD of all additive functions or the class HF of all Hamel functions.

Before formulating the appropriate theorem we recall what is known about values of the cardinal function A. (See Sections 1.2 and 3.2.)

$$
3 \leq \mathrm{A}(\mathrm{HF}) \leq \omega<\mathfrak{c}^{+}=\mathrm{A}(\mathrm{Ext}) \leq \mathrm{A}(\mathrm{AC})=\mathrm{A}(\mathrm{Conn})=\mathrm{A}(\mathrm{D}) \leq \mathrm{A}(\mathrm{PC})=2^{\mathfrak{c}}
$$

Theorem 4.3.1. Let $\mathcal{F} \in\{\mathrm{AD}, \mathrm{HF}\}$. The following holds.

(i) $\operatorname{Add}(\mathcal{F}, \mathcal{G})=\mathrm{A}(\mathcal{G})$ and $\operatorname{Add}(\mathcal{G}, \mathcal{F})=\mathrm{A}(\mathcal{F})$ for $\mathcal{G} \in\{$ Ext, AC, Conn, D, PC $\}$. In particular, $\operatorname{Add}(\mathcal{G}, \mathrm{AD})=\mathrm{A}(\mathrm{AD})=2$. In addition, we also have that $\operatorname{Add}(\mathrm{C}, \mathcal{F})=\operatorname{Add}(\mathcal{F}, \mathrm{C})=1=\operatorname{Add}(\mathrm{AD}, \mathrm{HF})=\operatorname{Add}(\mathrm{HF}, \mathrm{AD})$.

(ii) $\operatorname{Add}(\mathrm{SZ}, \mathcal{F})=\mathrm{A}(\mathcal{F})$ and $\operatorname{Add}(\mathcal{F}, \mathrm{SZ})>\mathfrak{c}$. 
Part (ii) of the theorem gives only a lower bound for the cardinals $\operatorname{Add}(\mathrm{AD}, \mathrm{SZ})$ and $\operatorname{Add}(\mathrm{HF}, \mathrm{SZ})$. It is unknown whether $\operatorname{Add}(\mathrm{AD}, \mathrm{SZ})=\operatorname{Add}(\mathrm{HF}, \mathrm{SZ})=\mathrm{A}(\mathrm{SZ})$.

Problem 4.3.2 Does $\operatorname{Add}(\mathcal{F}, \mathrm{SZ})$ equal to $\mathrm{A}(\mathrm{SZ})$ for $\mathcal{F} \in\{\mathrm{AD}, \mathrm{HF}\}$ ?

Before we proceed to the proof of Theorem 4.3.1 let us comment on how the class HF of Hamel functions relates to all the other families in terms of inclusion and intersection. Proposition 1.2.1 (iv) states that if $\operatorname{Add}\left(\mathcal{F}_{1}, \mathcal{F}_{2}\right) \geq 2$ then $\mathcal{F}_{1} \cap \mathcal{F}_{2} \neq \emptyset$. Thus, based on the values of Add given by the above theorem, we conclude that there exists a Hamel function belonging to each of Ext, AC, Conn, D, PC, or SZ. From Fact 3.2.3 we obtain $\mathrm{HF} \cap \mathrm{C}=\emptyset$. This implies that none of Ext, AC, Conn, D, or $\mathrm{PC}$ is contained in $\mathrm{HF}$. It is obvious that $\mathrm{SZ} \nsubseteq \mathrm{HF}$. But neither $\mathrm{HF}$ is contained in SZ. The latter holds because, based on Lemma 4.1.4, there is a Hamel function which is constant on a set of size $\mathfrak{c}$. So what is left to determine is whether HF is a subset of one of AC, Conn, D, or PC. Since all peripherally continuous functions contain all the other classes, it is sufficient to find out whether $\mathrm{HF} \subseteq \mathrm{PC}$. As one might expect, the inclusion $\mathrm{HF} \subseteq \mathrm{PC}$ is not true. An example of a Hamel function which is not peripherally continuous can be easily constructed by induction.

Example 4.3.3 There exists a Hamel function $h: \mathbb{R} \rightarrow \mathbb{R}$ which is not peripherally continuous.

Proof. See (o) in the proof of Theorem 4.3 .1 (ii).

Before we move to the proof of Theorem 4.3.1 we need one more lemma.

Lemma 4.3.4 $\operatorname{Add}(\mathcal{F}, \mathrm{D}) \geq \mathrm{A}(\mathrm{D}(\mathrm{P}))$ for $\mathcal{F}=\mathrm{AD}, \mathrm{HF}$. In particular, $\operatorname{Add}(\mathcal{F}, \mathrm{D})=$ $\mathrm{A}(\mathrm{D})$. 
Proof. The proof is done for the case $\mathcal{F}=\mathrm{AD}$. The argument for $\mathcal{F}=$ HF follows exactly the same path.

Let $P \subseteq \mathbb{R}$ be a perfect set with the property that $P \cup\{1\}$ is linearly independent over $\mathbb{Q}$. Observe that for every $p, q \in \mathbb{Q}, p \notin\{0,1\}$, we have $(p P+q) \cap P=\emptyset$. Now, consider a countable partition $\left\{P_{n}: n<\omega\right\}$ of $P$ into perfect sets. Using this partition and the above observation we can easily construct a family $\left\{P_{n}^{\star}: n<\omega\right\}$ of disjoint perfect sets such that $\bigcup_{n<\omega} P_{n}^{\star}$ is independent over $\mathbb{Q}$ and for every nontrivial interval $I \subseteq \mathbb{R}$ there is an $m<\omega$ such that $P_{m}^{\star} \subseteq I$. Note that $\bigcup_{n<\omega} P_{n}^{\star}$ is a $\mathfrak{c}$-dense meager $F_{\sigma}$-set.

To prove the inequality $\operatorname{Add}(\mathrm{AD}, \mathrm{D}) \geq \mathrm{A}(\mathrm{D}(\mathrm{P}))$ let us fix a family $F \subseteq \mathbb{R}^{\mathbb{R}}$ such that $|F|<\mathrm{A}(\mathrm{D}(\mathrm{P}))$. There exists a function $g \in \mathbb{R}^{\mathbb{R}}$ satisfying the property $g+F \subseteq \mathrm{D}(\mathrm{P})$. We claim that if $g^{\star}: \mathbb{R} \rightarrow \mathbb{R}$ is any additive extension of $g \mid \bigcup_{n<\omega} P_{n}^{\star}$ then $g^{\star}+F \subseteq$ D. More precisely, for every $f \in F, g^{\star}+f$ is strongly Darboux. To see this pick any $f \in F, y \in \mathbb{R}$, and any interval $I$. There exists $m<\omega$ such that $P_{m}^{\star}$ is contained in $I$. Furthermore, we can find $x \in P_{m}^{\star} \subseteq I$ for which $g^{\star}(x)+f(x)=g(x)+f(x)=y$. This shows that $g^{\star}+f$ is strongly Darboux.

The second statement in the lemma is proved by Proposition 4.1.5 and the inequality $\mathrm{A}(\mathrm{D}) \geq \operatorname{Add}(\mathrm{AD}, \mathrm{D}) \geq \mathrm{A}(\mathrm{D}(\mathrm{P}))$.

Proof of Theorem 4.3.1. (i) We start with showing $\operatorname{Add}(\mathcal{F}, \mathcal{G})=\mathrm{A}(\mathcal{G})$. Suppose that $\mathcal{G}=$ Ext. Since $\operatorname{Add}(\mathcal{F}$, Ext $) \leq \mathrm{A}($ Ext $)=\mathfrak{c}^{+}$for $\mathcal{F} \in\{\mathrm{AD}, \mathrm{HF}\}$, it suffices to show that $\operatorname{Add}(\mathcal{F}$, Ext $) \geq \mathfrak{c}^{+}$. So for every $F=\left\{f_{\xi}: \xi<\mathfrak{c}\right\} \subseteq \mathbb{R}^{\mathbb{R}}$ we need to find a $g \in \mathcal{F}$ such that $g+F \subseteq$ Ext.

Let $\left\langle D_{\xi}: \xi\langle\mathfrak{c}\rangle\right.$ be a sequence of pairwise disjoint $\mathfrak{c}$-dense meager $F_{\sigma}$ sets such that $\bigcup_{\xi<\mathfrak{c}} D_{\xi}$ is linearly independent over $\mathbb{Q}$. Such a sequence can be constructed 
in a similar way as the $\mathfrak{c}$-dense meager $F_{\sigma}$-set in the proof of Lemma 4.3.4. Now, by [4, Proposition 4.3], for every $\xi<\mathfrak{c}$ we can find $h_{\xi} \in$ Ext such that $\mathbb{R} \backslash D_{\xi}$ is $h_{\xi}$-negligible. Extend the partial function $\bigcup_{\xi<c}\left(h_{\xi}-f_{\xi}\right) \mid D_{\xi}$ to a function $g$ from $\mathcal{F}$. It is obvious that such $g$ exists in the case of additive functions. If $\mathcal{F}=\mathrm{HF}$ than the existence of $g$ follows from Lemma 4.1.4.

To see that $g+f_{\xi} \in$ Ext for every $\xi$, observe that $g+f_{\xi}=h_{\xi}$ on $D_{\xi}$. But the set $\mathbb{R} \backslash D_{\xi}$ is $h_{\xi}$-negligible. So each $g+f_{\xi}$ is extendable.

The equality $\operatorname{Add}(\mathcal{F}, \mathcal{G})=\mathrm{A}(\mathcal{G})$ for $\mathcal{G} \in\{\mathrm{AC}$, Conn, $\mathrm{D}\}$ follows from Propositions 4.1.2, 1.2.1 (1) and Lemma 4.3.4.

Now assume that $\mathcal{G}=\mathrm{PC}$. The proof of this part is similar to the proof of Theorem 2.1.1 (4). Fix a Hamel basis $H$ which is a Bernstein set. By choosing the sets $B_{\langle I, p, m\rangle}$ to be subsets of $H$, we can obtain, for a given family $F$ of real functions with cardinality less than $2^{\mathfrak{c}}$, a partial function $g^{\prime}: H \rightarrow \mathbb{R}$ such that for every $f \in F$, $g^{\prime}+f$ is dense in $\mathbb{R}^{2}$. Recall that a function with a dense graph is peripherally continuous. Thus, if $g: \mathbb{R} \rightarrow \mathbb{R}$ is an additive or Hamel function extending $g^{\prime}$ then $g+F \subseteq \mathrm{PC}$.

Next we show $\operatorname{Add}(\mathcal{G}, \mathcal{F})=\mathrm{A}(\mathcal{F})$. Let us first assume that $\mathcal{F}$ is the family of additive functions. Observe that $\mathrm{A}(\mathrm{AD})=2$. This follows from Proposition 1.2.1 (3) $\&(5)$ and obvious equality $\mathrm{AD}-\mathrm{AD}=\mathrm{AD}$. Next recall that $\operatorname{Add}(\mathcal{G}, \mathrm{AD}) \leq \mathrm{A}(\mathrm{AD})$ and $\mathcal{G}-\mathrm{AD}=\mathrm{AD}-\mathcal{G}=\mathcal{G}+\mathrm{AD}$ for all $\mathcal{G} \in\{$ Ext, $\mathrm{AC}$, Conn, D, PC $\}$. Thus, by Proposition 1.2.1 (3) and the equality $\operatorname{Add}(\mathrm{AD}, \mathcal{G})=\mathrm{A}(\mathcal{G})$ which is proved above, we conclude that $\mathcal{G}+\mathrm{AD}=\mathbb{R}^{\mathbb{R}}$. Consequently, $\operatorname{Add}(\mathcal{G}, \mathrm{AD})=2=\mathrm{A}(\mathrm{AD})$.

Now we consider case $\mathcal{F}=\mathrm{HF}$. Recall that the class Ext of extendable functions is contained in each of AC, Conn, D, or PC. So, by the monotonicity of Add, 
$\operatorname{Add}(\mathcal{G}, \mathrm{HF})=\mathrm{A}(\mathrm{HF})$ for $\mathcal{G} \in\{$ Ext, $\mathrm{AC}$, Conn, $\mathrm{D}, \mathrm{PC}\}$ will follow from the equality $\operatorname{Add}($ Ext, $\mathrm{HF})=\mathrm{A}(\mathrm{HF})$. Clearly, Add $($ Ext, HF $) \leq \mathrm{A}(\mathrm{HF})$. Let $F \subseteq \mathbb{R}^{\mathbb{R}}$ satisfies the condition that there is an $f \in \mathbb{R}^{\mathbb{R}}$ shifting $F$ into HF ( $f+F \subseteq$ HF.) Choose any $\mathfrak{c}$-dense $F_{\sigma}$ set $D$ independent over $\mathbb{Q}$. There is a function $h: D \rightarrow \mathbb{R}$ whose every extension onto $\mathbb{R}$ is in Ext (see proof of (ii).) Let $g \in \mathbb{R}^{\mathbb{R}}$ be an additive function extending $h-f$. Then we have $(g+f) \mid D=h$. So $g+f \in$ Ext. Since $f+F \subseteq$ HF, Fact 3.2.1 (i) implies that $(g+f)+F \in$ HF. This completes the proof of $\operatorname{Add}(\mathrm{Ext}, \mathrm{HF})=\mathrm{A}(\mathrm{HF})$.

What remains to prove is the last part of (i). The equality $\operatorname{Add}(\mathrm{C}, \mathrm{AD})=$ $\operatorname{Add}(\mathrm{AD}, \mathrm{C})=1$ is implied by Proposition 1.2.1 (3) and the fact $\mathrm{C}-\mathrm{AD}=\mathrm{AD}-\mathrm{C} \neq$ $\mathbb{R}^{\mathbb{R}}$. The characteristic function of a point, say $\chi_{\{0\}}$, is an example of a function witnessing the above property. Indeed, $\left(\chi_{\{0\}}+\mathrm{C}\right) \cap \mathrm{AD}=\emptyset$ because every additive function is either continuous or has a dense graph (see [3, Exercise 4, Section 7.3].)

To finish the proof of (i) we show that $\operatorname{Add}(\mathcal{H}, \mathrm{HF})=\operatorname{Add}(\mathrm{HF}, \mathcal{H})=1$ for $\mathcal{H}=\mathrm{AD}, \mathrm{C}$. But this follows easily from Proposition 1.2.1 (4) and $\mathcal{H} \cap \mathrm{HF}=\emptyset$ (see Fact 3.2.1 (iii).)

(ii) First we prove that $\operatorname{Add}(\mathcal{F}, \mathrm{SZ})>\mathfrak{c}$. Let us fix a family $F=\left\{h_{\xi}: \xi<\mathfrak{c}\right\} \subseteq \mathbb{R}^{\mathbb{R}}$. We will construct by induction a function $g \in \mathcal{F}$ satisfying $g+F \subseteq$ SZ. First we assume that $\mathcal{F}=\mathrm{AD}$.

Let $H=\left\{x_{\xi}: \xi<\mathfrak{c}\right\}$ be a Hamel basis $H=\left\{x_{\xi}: \xi<\mathfrak{c}\right\}$. We will define the function $g$ on $H$ and then extend it onto $\mathbb{R}$. For a given $\alpha<\mathfrak{c}$, we choose

$$
g\left(x_{\alpha}\right) \notin\left(\bigcup_{q \in \mathbb{Q}} \bigcup_{\xi, \gamma<\alpha} q\left(f_{\gamma}-h_{\xi}\right)\left[\operatorname{Lin}_{\mathbb{Q}}\left(x_{\beta}: \beta \leq \alpha\right)\right]\right)+g\left[\operatorname{Lin}_{\mathbb{Q}}\left(x_{\beta}: \beta<\alpha\right)\right],
$$

where $\left\langle f_{\alpha}: \alpha<\mathfrak{c}\right\rangle$ is a sequence of all continuous functions defined on $G_{\delta}$ subsets 
of $\mathbb{R}$. Such a choice is possible because the cardinality of the considered set is less than $\mathfrak{c}$. This choice also assures that $g+F \subseteq$ SZ. To see this observe the following: $\left[g+h_{\xi}=f_{\alpha}\right]=\left[g=f_{\alpha}-h_{\xi}\right] \subseteq \operatorname{Lin}_{\mathbb{Q}}\left(x_{\beta}: \beta \leq \max (\alpha, \xi)\right)$ for all $\alpha, \xi<\mathfrak{c}$. Thus $\left|\left[g+h_{\xi}=f_{\alpha}\right]\right| \leq \omega \max (\alpha, \xi)<\mathfrak{c}$, which proves that $g+h_{\xi} \in \mathrm{SZ}$.

Now consider the case $\mathcal{F}=\mathrm{HF}$ and fix an enumeration $\left\{x_{\xi}: \xi<\mathfrak{c}\right\}$ of $\mathbb{R}$. We will define an increasing sequence of partial functions $\left\langle g_{\xi}: \xi<\mathfrak{c}\right\rangle$ satisfying for all $\beta, \gamma, \xi<\mathfrak{c}$

(a) $x_{\xi} \in \operatorname{dom}\left(g_{\xi}\right),\left\langle 0, x_{\xi}\right\rangle \in \operatorname{Lin}_{\mathbb{Q}}\left(g_{\xi}\right)$, and $g_{\xi}$ is linearly independent,

(b) $\left|\operatorname{dom}\left(g_{\xi}\right)\right| \leq \max (\omega, \xi)$ and $\left[\left(g_{\xi}+h_{\beta}\right)=f_{\gamma}\right] \subseteq \operatorname{dom}\left(g_{\max (\beta, \gamma)}\right)$.

Put $g_{0}=\left\{\left\langle x_{0}, 1\right\rangle\right\}$. Clearly, $g_{0}$ satisfies all the required conditions.

Now suppose that $\alpha<\mathfrak{c}$, the sequence $\left\langle g_{\xi}: \xi<\alpha\right\rangle$ is already defined, and $\left\langle 0, x_{\alpha}\right\rangle \notin$ $\operatorname{Lin}_{\mathbb{Q}}\left(\bigcup_{\xi<\alpha} g_{\xi}\right)$. Choose an $x \notin \operatorname{Lin}_{\mathbb{Q}}\left(\bigcup_{\xi<\alpha} \operatorname{dom}\left(g_{\xi}\right)\right)$. We will define $g_{\alpha}$ as an extension of $\bigcup_{\xi<\alpha} g_{\xi}$ onto $\left\{-x, x, x_{\alpha}\right\} \cup \bigcup_{\xi<\alpha} \operatorname{dom}\left(g_{\xi}\right)$. Let $g_{\alpha}(-x)$ and $g_{\alpha}(x)$ be such that $g_{\alpha}(-x)+g_{\alpha}(x)=x_{\alpha}$ and

$$
g_{\alpha}(-x), g_{\alpha}(x) \notin\left(\bigcup_{\beta, \gamma<\alpha}\left(f_{\gamma}-h_{\beta}\right)\left[\{-x, x\} \cup \bigcup_{\xi<\alpha} \operatorname{dom}\left(g_{\xi}\right)\right]\right) .
$$

We can easily define $g_{\alpha}$ at $x_{\alpha}$ if necessary, preserving the conditions (a) and (b). This completes the construction of $\left\langle g_{\xi}: \xi<\mathfrak{c}\right\rangle$.

Put $g=\bigcup_{\xi<\mathfrak{c}} g_{\xi}$. It is easily seen that $g+F \subseteq$ SZ. Since $\{0\} \times \mathbb{R} \subseteq \operatorname{Lin}_{\mathbb{Q}}(g)$ we also have that $\mathbb{R}^{2} \subseteq \operatorname{Lin}_{\mathbb{Q}}(g)$. By (a) $g$ is linearly independent so $g \in \mathrm{HF}$.

Let us notice that $g$ could be constructed in such way that its graph has an isolated point. This shows, in particular, that

(o) there is a Hamel function which is not peripherally continuous. 
This is so because if a function $f: \mathbb{R} \rightarrow \mathbb{R}$ is peripherally continuous then for every $x$ there exist sequences $a_{n} \nearrow x$ and $b_{n} \searrow x$ such that $f\left(a_{n}\right), f\left(b_{n}\right) \rightarrow f(x)$.

Next we show that $\operatorname{Add}(\mathrm{SZ}, \mathcal{F})=\mathrm{A}(\mathcal{F})$. To prove this equality for $\mathcal{F}=$ HF fix a $G \subseteq \mathbb{R}^{\mathbb{R}}$ such that $|G|<\mathrm{A}(\mathrm{HF})$. There exists a $g \in \mathbb{R}^{\mathbb{R}}$ with the property that $g+G \subseteq \mathrm{HF}$. From the inequality $\operatorname{Add}(\mathrm{AD}, \mathrm{SZ})>\mathfrak{c}$, which has been proved, we conclude that $f+g \in \mathrm{SZ}$ for some $f \in \mathrm{AD}$. But, by Fact 3.2.1 (i), $(f+g)+G=$ $f+(g+G) \subseteq \mathrm{HF}$. This shows that $\mathrm{Add}(\mathrm{SZ}, \mathrm{HF}) \geq \mathrm{A}(\mathrm{HF})$. The opposite inequality is obvious.

To see $\operatorname{Add}(\mathrm{SZ}, \mathrm{AD})=\mathrm{A}(\mathrm{AD})$ recall that, based on $(\mathrm{i}), \mathrm{A}(\mathrm{AD})=2$. By the monotonicity of Add we get that $\operatorname{Add}(\mathrm{SZ}, \mathrm{AD}) \leq \mathrm{A}(\mathrm{AD})$. In the previous part of this proof we have shown that $\operatorname{Add}(\mathrm{HF}, \mathrm{SZ})>\mathfrak{c}$. Thus, by Proposition 1.2.1 (3) we obtain $\operatorname{Add}(\mathrm{SZ}, \mathrm{HF}) \geq 2=\mathrm{A}(\mathrm{AD})$. Consequently, $\mathrm{Add}(\mathrm{SZ}, \mathrm{AD})=\mathrm{A}(\mathrm{AD})$. 


\section{Bibliography}

[1] M. Balcerzak, K. Ciesielski, T. Natkaniec, Sierpiński-Zygmund functions that are Darboux, almost continuous, or have a perfect road, Arch. Math. Logic 37 (1997), 29-35.

[2] A.L Cauchy Cours d'analyse de l'Ecole Polytechnique, 1. Analyse algébrique, V., Paris, 1821 [Oeuvres (2) 3, Paris, 1897].

[3] K. Ciesielski, Set Theory for the Working Mathematician, London Math. Soc. Student Texts 39, Cambridge Univ. Press 1997.

[4] K. Ciesielski, J. Jastrzȩbski, Darboux-like functions within the classes of Baire one, Baire two, and additive functions, Topology Appl. 103 (2000), 203-219.

[5] K. Ciesielski, A.W. Miller Cardinal invariants concerning functions whose sum is almost continuous, Real Anal. Exchange 20 (1994-95), 657-672.

[6] K. Ciesielski, T. Natkaniec, Algebraic properties of the class of SierpinskiZygmund functions, Topology Appl. 79 (1997), 75-99.

[7] K. Ciesielski, I. Recław, Cardinal invariants concerning extendable and peripherally continuous functions, Real Anal. Exchange 21 (1995-96), 459-472. 
[8] G. Darboux, Memoire sur les fonctions discontinues, Ann. Sci. Scuola Norm. Sup. 4 (1875), 57-112.

[9] R.G. Gibson, T. Natkaniec, Darboux-like functions, Real Anal. Exchange 22 (2) (1996-97), 492-533.

[10] G. Hamel, Eine Basis aller Zahlen und die unstetigen Lösungen der Funktionalgleichung $f(x+y)=f(x)+f(y)$, Math. Ann. 60 (1905), 459-462.

[11] W. Hurewicz and H. Wallman, Dimension Theory, Princeton University Press, 1948.

[12] F. Jordan, Sums of Darboux-like functions from $\mathbb{R}^{n}$ to $\mathbb{R}^{m}$, Real Anal. Exchange 24 (2) (1998-99), 729-759.

[13] A. Kamburelis, On cardinal numbers related to Baire property, Ph.D. Dissertation, Wrocław 1989.

[14] K.R. Kellum, Sums and limits of almost continuous functions, Colloq. Math. 31 (1974), 125-128.

[15] K.R. Kellum, Almost Continuity and connectivity - sometimes it's as easy as to prove a stronger result, Real Anal. Exchange 8 (1982-83), 244-252.

[16] M. Kuczma, An Itroduction to the Theory of Functional Equations and Inequalities, Polish Scientific Publishers, PWN, Warszawa, 1985.

[17] K. Kuratowski, Topologie I, Warszawa 1958.

[18] T. Natkaniec, Almost Continuity, Real Anal. Exchange 17 (1991-92), 462-520. 
[19] J.C. Oxtoby, Measure and Category, Springer-Verlag, Amsterdam 1971.

[20] K. Płotka, Sum of Sierpiński-Zygmund and Darboux Like functions, Topology Appl., to appear.

[21] K. Płotka, On real functions defined on $\mathbb{R}$ whose graph is a Hamel basis, to be submitted.

[22] K. Płotka, Sierpiński-Zygmund sets on the plane, to be submitted.

[23] W. Sierpiński, A. Zygmund, Sur une fonction qui est discontinue sur tout ensemble de puissance du continu, Fund. Math 4 (1923), 316-318. 